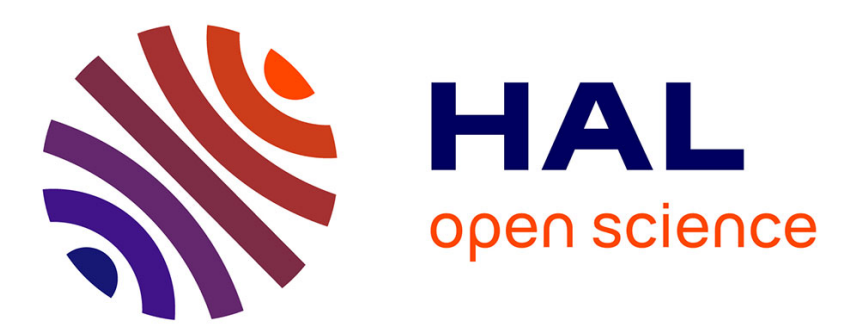

\title{
Twins and allotwins of basicmica polytypes: theoretical derivation and identification in the reciprocal space
}

\author{
Massimo Nespolo, Giovanni Ferraris, Hiroshi . Takeda
}

\section{To cite this version:}

Massimo Nespolo, Giovanni Ferraris, Hiroshi . Takeda. Twins and allotwins of basicmica polytypes: theoretical derivation and identification in the reciprocal space. Acta Crystallographica Section A : Foundations and Advances [2014-..], 2000, A56, pp.132-148. 10.1107/S0108767399014907. hal00130642

\section{HAL Id: hal-00130642 \\ https://hal.science/hal-00130642}

Submitted on 13 Feb 2007

HAL is a multi-disciplinary open access archive for the deposit and dissemination of scientific research documents, whether they are published or not. The documents may come from teaching and research institutions in France or abroad, or from public or private research centers.
L'archive ouverte pluridisciplinaire HAL, est destinée au dépôt et à la diffusion de documents scientifiques de niveau recherche, publiés ou non, émanant des établissements d'enseignement et de recherche français ou étrangers, des laboratoires publics ou privés. 
Acta Crystallographica Section A

Foundations of

Crystallography

ISSN 0108-7673

\section{Twins and allotwins of basic mica polytypes: theoretical derivation and identification in the reciprocal space}

Massimo Nespolo, Giovanni Ferraris and Hiroshi Takeda

Copyright (C) International Union of Crystallography

Author(s) of this paper may load this reprint on their own web site provided that this cover page is retained. Republication of this article or its storage in electronic databases or the like is not permitted without prior permission in writing from the IUCr. 
Acta Crystallographica Section A

Foundations of Crystallography

ISSN 0108-7673

Received 14 September 1999

Accepted 19 November 1999

(C) 2000 International Union of Crystallography Printed in Great Britain - all rights reserved

\section{Twins and allotwins of basic mica polytypes: theoretical derivation and identification in the reciprocal space}

\author{
Massimo Nespolo, ${ }^{\mathrm{a} *}$ Giovanni Ferraris $^{\mathrm{b}}$ and Hiroshi Takeda ${ }^{\mathrm{c}}$

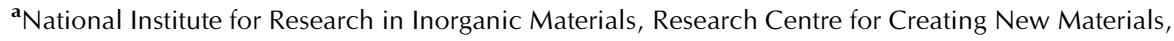 \\ 1-1 Namiki, Tsukuba, 305-0044 Ibaraki, Japan, ${ }^{\mathbf{b}}$ University of Torino, Department of Mineralogical \\ and Petrologic Sciences, via Valperga Caluso 35, 10125 Torino, Italy, and ${ }^{\mathbf{C}}$ University of the \\ Ryukyus, Faculty of Science, Department of Physics and Earth Sciences, 1-Senbaru, Nishihara, \\ Nakagami-Gun, 903-0213 Okinawa, Japan. Correspondence e-mail: nespolo@nirim.go.jp
}

The geometry of the diffraction pattern from twins and allotwins of the four basic mica polytypes $\left(1 M, 2 M_{1}, 3 T, 2 M_{2}\right)$ is analysed in terms of the 'minimal rhombus', a geometrical asymmetric unit in reciprocal space defined by nine translationally independent reciprocal-lattice rows. The minimal rhombus contains the necessary information to decompose the reciprocal lattice of twins or allotwins into the reciprocal lattices of the individuals. The nine translationally independent reciprocal-lattice rows are divided into three types ( $S, D$ and $X$ ): rows of different type are not overlapped by the $n \times 60^{\circ}$ rotations about $\mathbf{c}^{*}$, which correspond to the relative rotations between pairs of twinned or allotwinned individuals. A symbolic representation of the absolute orientation of the individuals, similar to that used for layers in polytypes, is introduced. The polytypes $1 M$ and $2 M_{1}$ undergo twinning by reticular pseudo-merohedry with five pairs of twin laws: they produce twelve independent twins, of which nine can be distinguished by the minimal rhombus analysis. The $2 M_{2}$ polytype has two pairs of twin laws by pseudo-merohedry, which give a single diffraction pattern geometrically indistinguishable from that of the single crystal, and three pairs of twin laws by reticular pseudo-merohedry, which give a single diffraction pattern different from that of the single crystal. The $3 T$ polytype has three twin laws: one corresponds to complete merohedry and the other two to selective merohedry. Selective merohedry produces only partial restoration of the weighted reciprocal lattice built on the family rows and the presence of twinning can be recognized from the geometry of the diffraction pattern.

\section{Introduction}

Micas, through their polytypes, give one of the most complex series of inorganic structures known to date (Smith \& Yoder, 1956; Ross et al., 1966; Takeda, 1967; Rieder, 1970; Takeda \& Ross, 1995; Nespolo \& Takeda, 1999; Kogure \& Nespolo, 1999). Micas often form twins but the presence of twinning is not always evident through a morphological analysis. Oriented crystal associations of different polytypes with the same chemical composition (allotwins: Nespolo, Kogure \& Ferraris, 1999) or with different chemical compositions (epitaxy/ syntaxy) have also been reported (e.g. Babu, 1969; Rieder, 1970; Gresens \& Stensrud, 1971; Tatekawa, 1975; Nespolo, Kogure \& Ferraris, 1999).

Takano \& Takano (1958) introduced the definitions of 'real polytypism' and 'apparent polytypism' to distinguish true polytypes from twins that can be mistaken for polytypes. Geometrical criteria for distinguishing between real and apparent polytypism have recently been developed (Nespolo, 1999a) and applied to reinvestigate a previous finding of a long-period polytype of biotite (Nespolo \& Takeda, 1999). The purpose of the present research is to obtain general criteria to decompose the composite weighted reciprocal lattice (w.r.l.) of a mica twin or allotwin, as it appears in the diffraction pattern, into the w.r.l. of the individuals, ${ }^{1}$ obtaining the relative rotations between individuals (preliminary account in Nespolo, Takeda, Ferraris \& Kogure, 1997). Since more than one law may correspond to the same orientation of the individuals, the identification of the twin or allotwin laws requires a morphological analysis.

Micas are built by a layer ( $M$ layer) $[C 12 / m(1)]$ in which an octahedral $(O)$ sheet $[P(\overline{3}) 1 \mathrm{~m}]$ is sandwiched between two

\footnotetext{
1 The term 'individual' is here used to indicate one crystal in a twin and the term 'single crystal' to mean an untwinned crystal.
} 
tetrahedral $(T)$ sheets [P(6)mm] (Pabst, 1955; Ďurovič, 1994a) [layer-group notation after Dornberger-Schiff (1959); details given by Merlino (1990)]. In the $O$ sheet, three translationally independent cation sites exist: the M1 site, on the mirror plane, and a pair of M2 sites, related by the mirror plane (Fig. 1a). Cleavage normally occurs between neighbouring $M$ layers (Kogure, 1997). Several structural distortions are present in the real layer (Abbott \& Burnham, 1988), which however mainly influence the diffraction intensities and much less the geometry of the diffraction pattern (Takeda \& Ross, 1995; Nespolo, 1999a). The so-called 'Pauling model' (Pauling, 1930) neglects all the distortions, the most relevant of which is the ditrigonal rotation of the tetrahedra, which reduces the symmetry of the $T$ sheets to $P(3) 1 m$ (Fig. 1a) and is described by the so-called 'trigonal model' (Nespolo, Takeda, Kogure \& Ferraris, 1999).

The thickness of the unit layer corresponds to the period along $\mathbf{c}$ for the $1 M$ polytype $\left(c_{1 M} \approx 10 \AA\right)$. The vertical distance between two closest interlayer cations is $c_{0}=c_{1 M} \sin \beta_{1 M}=1 / c_{1}^{*}$ (Backhaus \& Durovič, 1984; Nespolo, 1999a), where $c_{1}^{*}$ (about $0.1 \AA^{-1}$ ) is the reciprocal-space period along $\mathbf{c}^{*}$ for $1 M$. In the diffraction pattern of an $N$-layer polytype, $N$ reflections appear in the $c_{1}^{*}$ repeat along some reciprocal-lattice rows (nonfamily rows: see below) parallel to c. $^{*}$.

All mica polytypes have an (ideally) orthohexagonal axial setting based on two (pseudo)orthohexagonal axes $(a, b)$ in the plane of the layer [(001) plane] (Figs. $1 a$ and $1 b)$, for which the relation

$$
b=a 3^{1 / 2}, \quad \gamma=90^{\circ}
$$

is approximately obeyed. The $a$ and $b$ axes define the two shortest mutually orthogonal translations in the plane of the layer. A (pseudo)orthohexagonal setting based on $(a, b)$ axes can be built, with period along $\mathbf{c}$ equal to $N^{\prime} c_{0}$, where

$$
\begin{aligned}
& \text { orthogonal polytypes : } \quad N^{\prime}=N \\
& \text { non-orthogonal polytypes : } \quad N^{\prime}=3 N .
\end{aligned}
$$

This setting is labelled $C_{1}$ (Arnold, 1996; Nespolo, Takeda \& Ferraris, 1997; Nespolo et al., 1998) ${ }^{2}$ and its cell coincides with the conventional cell of orthogonal polytypes, whereas it contains three conventional cells in the case of non-orthogonal polytypes. The $C_{1}$ setting is taken to coincide with Zvyagin's orthohexagonal setting (Zvyagin, 1997) and thus its $a$ and $b$ axes are directed up and left, respectively, in the plane of the drawing (Fig. 1b). All the reflection conditions and the definitions in reciprocal space given below implicitly include the $C$ centring condition $h+k=0(\bmod 2)$.

Among several kinds of symbols that have been introduced to describe mica polytypes (see Nespolo, Takeda, Kogure \& Ferraris, 1999), those giving the absolute orientation and relative displacement of $M$ layers are hereinafter used to derive a similar symbolism for twins. According to Zvyagin (1997), the whole $M$ layer is described through a shortened

\footnotetext{
${ }^{2}$ Some authors, such as the OD school, adopted the $C_{2}$ cell, which has the $a$ axis bisecting the hexagonal $a_{1}$ and $a_{2}$ axes (e.g. Durovič et al., 1984).
}

symbolism $I J K \ldots$, where $I, J, K, \ldots$ can take six integral values ( 1 to 6 ) (Fig. $1 b$ ). These symbols have been called $Z$ symbols (Nespolo, Takeda \& Ferraris, 1997); their parity (odd or even) is called 'orientation parity' (Zvyagin, 1997). Z symbols indicate the absolute orientation of the (001) projection of the $\mathbf{Z}$ vectors, i.e. the vectors connecting two interlayer cations on the opposite sides of an $M$ layer and passing through the origin of the $O$ sheet, oriented along $+\mathbf{c}$ (Fig. 2). ${ }^{3}$

Two types of classification of mica polytypes are necessary to develop general criteria for the interpretation of the composite diffraction pattern from mica twins. The OD classification (Backhaus \& Ďurovič, 1984; Nespolo, 1999a) distinguishes polytypes on the basis of the equivalence of layer pairs. The reticular classification (Nespolo, Takeda \& Ferraris, 1997; Nespolo et al., 1998) emphasizes the metric relations in both direct and reciprocal spaces. A summary of these classifications is given below.

\subsection{OD classification of mica polytypes}

All polytypes of a substance built on the same structural principle belong to the same family and share a common fictitious structure, called family structure, in which all the possible positions of all OD layers are simultaneously realized (Durovič, 1994b). The symmetry and basis vectors of the family structure are obtained by completing the local symmetry operations of a space groupoid of any member of the family, to global symmetry operations of a space group (Fichtner, 1977). ${ }^{4}$ The Fourier transform of the family structure corresponds to a three-dimensional subset of reflections, called family reflections (Dornberger-Schiff \& Fichtner, 1972), which are always sharp and are common to all polytypes of the same family. The remaining ones are called nonfamily reflections and are typical of each polytype; they can be sharp or diffuse, depending whether the polytype is ordered or not (Ďurovič \& Weiss, 1986; Ďurovič, 1997, 1999). The family reflections correspond to a subgroup of translations of the polytype reciprocal lattice, whereas the basis vectors of the family structure correspond to a supergroup of translations of the polytype direct lattice. The lattices corresponding to the family structure are thus a superlattice in direct space and a sublattice in reciprocal space. ${ }^{5}$ Family rows are called the reciprocal-lattice rows corresponding to family reflections.

\footnotetext{
${ }^{3}$ In the most general case, $\mathbf{Z}$ vectors are defined by pairs of vectors describing half-layers. Within the so-called homo-octahedral approximation (Nespolo, Takeda, Kogure \& Ferraris, 1999), which assumes the origin of the $O$ sheet at the M1 site, the two halves of a layer have the same orientation and thus the above shortened symbolism is adopted. For details, see Zvyagin (1997).

${ }^{4}$ A global (also called total) operation is an ordinary space-group operation, i.e. a symmetry operation valid in the whole crystal space. A local operation is a space-groupoid operation, i.e. a symmetry operation valid in a subspace of the crystal space. A partial operation is a nonsymmetry operation valid in a subspace of the crystal space (Sadanaga et al., 1980).

${ }^{5}$ Since the lattice obtained from the original one by taking a subgroup (supergroup) of translations has a larger (smaller) unit cell, in some publications (e.g. Santoro \& Mighell, 1972; Giacovazzo, 1992; Nespolo, Takeda, Kogure \& Ferraris, 1999), the terms superlattice and sublattice are defined in the opposite way. Here we adopt the definition in terms of the group-subgroup relations, in agreement with International Tables for Crystallography, Vol. A, 5th ed. (Th. Hahn, personal communication).
} 
For micas, within the Pauling model, the family reflections correspond to $h=0(\bmod 3)$ and $k=0(\bmod 3)$ and are common to all polytypes (Durovič et al., 1984; Weiss \& Wiewióra, 1986). Reflections in the $c_{1}^{*}$ repeat obey the presence criterion $l=0\left(\bmod N^{\prime}\right)$, where $N^{\prime}$ is defined in equation (2). The family structure is ninefold (the subgroup of translations in the reciprocal space has order 9) and has symmetry $P 6 / \mathrm{mmm}$. Within the trigonal model, the family reflections correspond to $k=0(\bmod 3)$ and the family structure is threefold (the subgroup of translations in the reciprocal

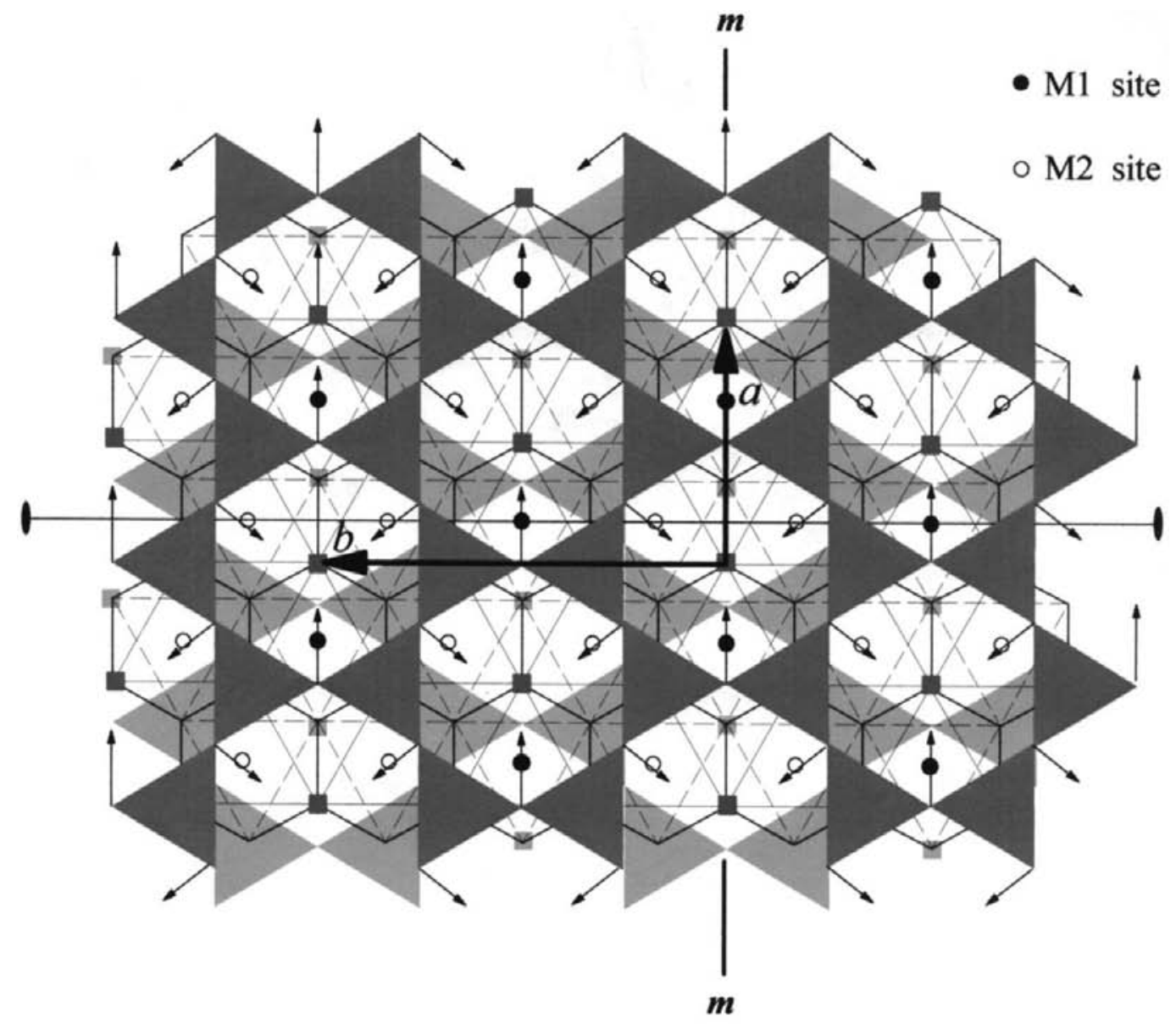

(a)

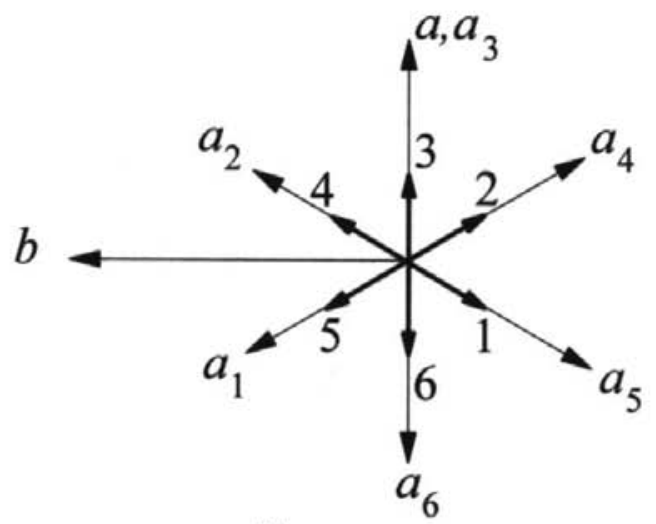

(b)

Figure 1

(a) Projection of the $M$ layer onto the (001) plane. Dark- and light-grey $T$ sheets are above and below the $O$ sheet, respectively. Dark- and light-grey squares represent interlayer cations above and below the $M$ layer, respectively. M1 sites are shown by black circles. Thin arrows indicate the direction of the ditrigonal rotation (shown only for the upper $T$ sheet). The independent symmetry elements (twofold rotation axis and symmetry plane) of the $2 / m$ point group are shown. $(b)$ Axes $(a, b)$ of the space-fixed reference $\left(C_{1}\right.$ setting $)$ and of the structure-related references in the six possible orientations $\left(a_{1}-a_{6}\right)$ and corresponding $Z$ symbols. The $c$ axis of the $C_{1}$ setting is perpendicular to the layer. The six axes $a_{1}-a_{6}$ indicate the six possible directions of the structure-related references, and thus the six possible orientations of the layer $\left(b_{1}-b_{6}\right.$ axes are not shown). The direction of the intralayer displacement is indicated by the $Z$ symbol $i(i=1-6)$ when the $a_{i}$ axis is parallel to the space-fixed $a$ axis (modified after Zvyagin, 1997). 
space has order 3). Family reflections for the threefold structure are no longer common to all polytypes but depend upon the relative rotations between successive layers. Within the trigonal model, mica polytypes are thus classified in the following three kinds:

(i) Subfamily A polytypes: successive layers are rotated by $2 n \times 60^{\circ}$ only and are described by $Z$ symbols of the same parity. The threefold family structure has symmetry $H_{R} \overline{3} 1 m$ (the subscript $R$ indicates that the smallest cell is rhombohedral). Reciprocal-lattice rows corresponding to $h \neq 0(\bmod 3)$ and $k=0(\bmod 3)$ are family rows and show a single reflection in the $c_{1}^{*}$ repeat, obeying the presence criterion $l_{C_{1}}=\left(N^{\prime} h / 3\right)\left(\bmod N^{\prime}\right)$ if the parity of layers is odd (obverse setting of the family structure); instead, $l_{C_{1}}=\left(2 N^{\prime} h / 3\right)\left(\bmod N^{\prime}\right)$ if the parity of layers is even (reverse setting of the family structure).

(ii) Subfamily B polytypes: successive layers are rotated by $(2 n+1) \times 60^{\circ}$ only and are described by $Z$ symbols of alternating parity. The threefold family structure has symmetry $\mathrm{H}_{3} / \mathrm{mcm}$. Reciprocal-lattice rows corresponding to $h \neq 0(\bmod 3)$ and $k=0(\bmod 3)$ are family rows and show two reflections in the $c_{1}^{*}$ repeat, with the presence criterion $l_{C_{1}}=0\left(\bmod N^{\prime} / 2\right)$.

(iii) Mixed-rotation polytypes: successive layers are rotated by both $2 n \times 60^{\circ}$ and $(2 n+1) \times 60^{\circ}$ and are described by $Z$ symbols of mixed nonalternating parity; the only family rows are those corresponding to both $h$ and $k=0(\bmod 3)$. The only family structure is the ninefold one.

The period along $\mathbf{c}$ of the family structure is $c_{0}$ in the Pauling model, whereas in the trigonal model it is $3 c_{0}$ (subfamily $A$ ) or $2 c_{0}$ (subfamily B) (Ďurovič et al., 1984).

\subsection{Reticular classification of mica polytypes}

The reticular classification of mica polytypes is based upon the projection $\mathbf{c}_{n}$ of the $c$ axis onto the (001) plane. Mica polytypes are divided into three kinds:

(i) orthogonal polytypes: $\mathbf{c}_{n}=(0,0)$;

(ii) Class a polytypes: $\mathbf{c}_{n}=(-1 / 3,0)$;

(iii) Class $b$ polytypes: $\mathbf{c}_{n}=(0,-1 / 3)$.

The number of layers $N$ in a mica polytype is suitably expressed as:

$$
N=3^{n}(3 K+L)
$$

where $n$ defines the Series, $L$ the Subclass and $K$ is an integer that appears in the transformation matrices relating different axial settings ( $n \geq 0, L=1,2, K \geq 0)$.

The geometry of the diffraction pattern of a twinned mica polytype belonging to Series $n$ is often similar to that of an untwinned mica polytype belonging to Series $n+1$ (Nespolo, 1999a). Therefore, when the diffraction pattern of a mica crystal shows $3 p$ equally spaced reflections in the $c_{1}^{*}$ repeat, the possibility of apparent polytypism must be taken into account. The derivation of the possible twin diffraction patterns is thus a prerequisite for the study of polytypism.

The present geometrical analysis of mica twins and allotwins is devoted to the four most common polytypes, namely $1 M(Z=3$; subfamily $A$, Class a, Series 0$), 2 M_{1}(Z=24$; subfamily $A$, Class a, Series 0$), 3 T(Z=351$ or 315 ; subfamily $A$, orthogonal, Series 1$)$ and $2 M_{2}(Z=45$; subfamily $B$, Class $b$, Series 0$)$. These are by far the most common polytypes and are called basic mica polytypes because the stacking sequence of longer-period polytypes is usually based on one of them

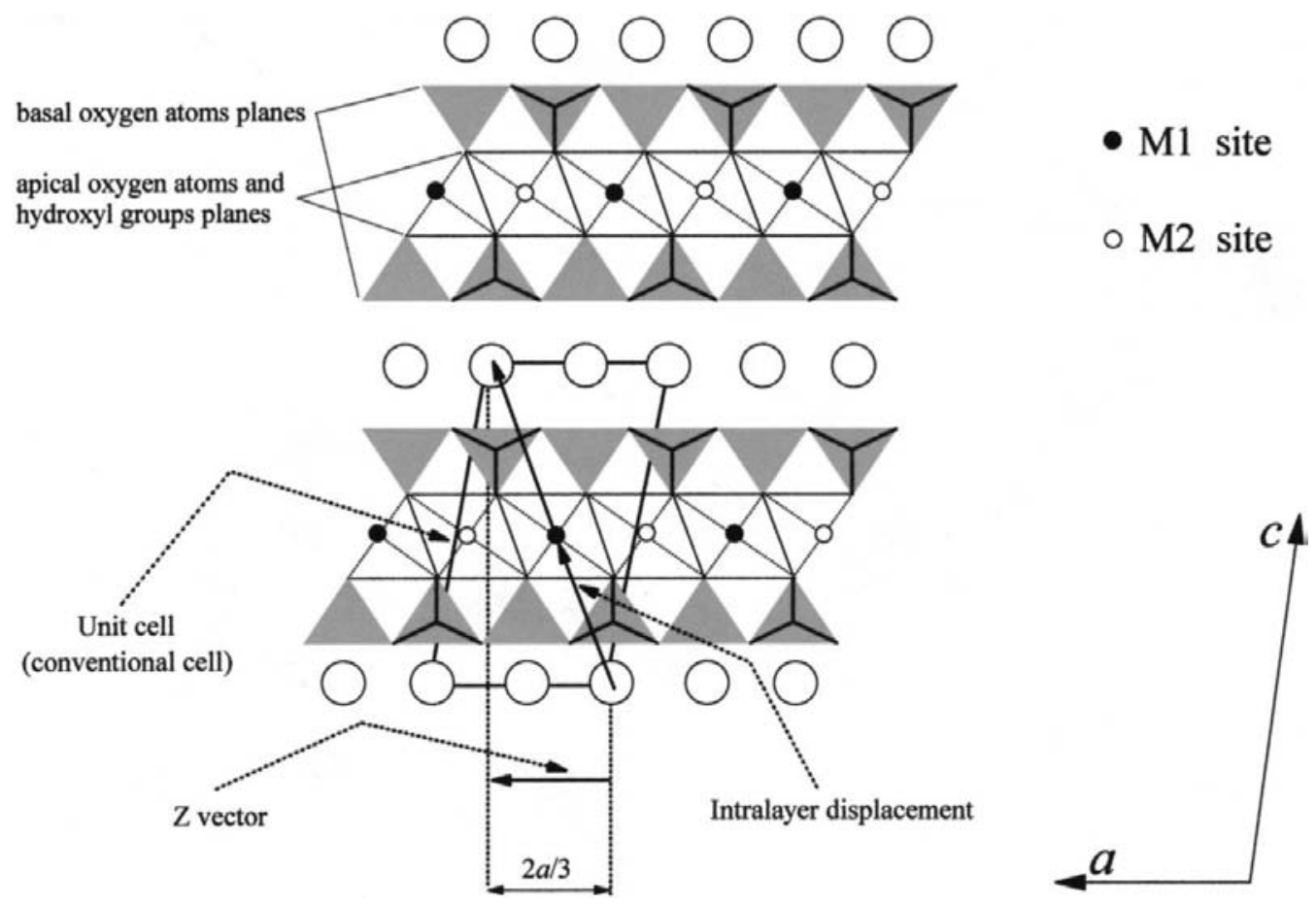

Figure 2

Projection along the $b$ axis of the $1 M$ mica structure, showing two layers with the same orientation. M1 and M2 cationic sites in the $O$ sheet occupy crystallographically distinct positions (cf. Fig. 1). The scale along $\mathbf{c}$ is compressed (modified after Nespolo, 1999b). 
(Baronnet \& Kang, 1989). The general approach derived henceforth can however be easily generalized to other polytypes.

\section{Twin laws for mica polytypes}

The basis for the derivation of the twin laws for mica polytypes in the general case is given in Nespolo (1999a). Here, the treatment is focused onto the four basic polytypes, and the derivation is performed through coset decomposition (Giacovazzo, 1992). Rotations in direct and reciprocal space are taken counterclockwise and clockwise, respectively. Symbols for the crystal systems of polytypes are given according to Guinier et al. (1984). In the case of twinning by merohedry, the twin laws are expressed through Shubnikov's two-colour group notation (the twin elements are dashed: Curien \& Le Corre, 1958). Twins in which two or more twin laws have operated are called composite twins (Nespolo, Takeda, Ferraris \& Kogure, 1997). The complete twin (Curien \& Donnay, 1959) is a twin by merohedry or reticular merohedry in which the number of individuals generated from the original one is equal to the number of possible twin laws.

Mica polytypes can belong to 24 space groups of five crystal systems (Takeda, 1971): $A, M, O, T$ and $H$. The pair of (pseudo)orthohexagonal axes $(a, b)$ in the plane of the layer is common to all polytypes. All polytypes admit a hexagonal twin lattice based on a pair of orthohexagonal axes $\left(a_{H}, b_{H}\right)$ parallel to $(a, b)$ : its symmetry corresponds to that of the ninefold family structure, namely $P 6 / \mathrm{mmm}$ (DornbergerSchiff et al., 1982; Backhaus \& Durovič, 1984). The cell of the twin lattice coincides with the $C_{1}$ cell for hexagonal and trigonal polytypes, and in this case a hexagonal cell can be chosen as well (Fig. 3). For all the others, equation (1) is obeyed by $\left(a_{H}, b_{H}\right)$ but only approximately by $(a, b)$. The angular $(\varepsilon)$ (Donnay et al., 1964) and the linear $(\eta)$ (Zvyagin \& Drits, 1996) deviations from the orthohexagonality (Fig. 4a) are related to the twin obliquity. Moreover, for non-orthogonal polytypes, the length of the $\mathbf{c}_{n}$ projection (\$1.2) actually deviates from $|a| / 3$ or $|b| / 3$ by a quantity that depends mainly upon the crystal chemistry. Rieder (1970) described this deviation in terms of the monoclinic angle and showed that it is not negligible for dioctahedral and $\mathrm{Li}-\mathrm{Fe}$ trioctahedral micas. The practical consequence is that the $C_{1}$ cell is no longer rigorously orthogonal and the reflections from twinned individuals may have a zigzag splitting around the position of the ideal row. This clearly indicates the presence of twinning. The relative rotations between individuals can be obtained also in this case by applying the geometrical treatment developed hereafter, provided the small splitting is neglected.

\subsection{Monoclinic holohedral polytypes}

Three of the four basic polytypes are monoclinic holohedral ( $1 M: C 2 / m ; 2 M_{1}$ and $2 M_{2}: C 2 / c$ ) and the $C_{1}$ cell contains three conventional cells. For $1 M$ and $2 M_{1}$ polytypes (Class $a$ ), twinning is by reticular pseudo-merohedry, with twin index 3 . Lattice nodes are completely restored by the twin operation in one plane out of three along $\mathbf{c}^{*}$, whereas they are not restored at all in the remaining two planes. The lattice of Class $b$ polytypes, to which $2 M_{2}$ belongs, admits a primitive cell close to rhombohedral (Nespolo, 1999a) and the classification of twin laws is based on this pseudo-symmetry. Point group $2 / m$ is a subgroup of order 6 of $6 / \mathrm{mmm}$, and thus six cosets can be obtained, but the unique axes do not coincide in the crystal lattice ( $b$ unique for Class $a$ polytypes; $a$ unique for Class $b$ polytypes) and in the twin lattice ( $c$ unique). Following Donnay et al. (1964), the coset decomposition is here given through left cosets. Results are expressed in the $C_{1}$ setting. Class a:

$$
\begin{aligned}
6 / m m m= & \left\{1,2_{[010]}, \overline{1}_{,} m_{(010)}\right\} \cup\left\{6_{[001]}^{-}, 2_{[110]}, \overline{6}_{[001]}^{-}, m_{(130)}\right\} \\
& \cup\left\{3_{[001]}^{-}, 2_{[310]}, \overline{3}_{[001]}^{-}, m_{(110)}\right\} \\
& \cup\left\{2_{[001]}, 2_{[100]}, m_{(001)}, m_{(100)}\right\} \\
& \cup\left\{3_{[001]}^{+}, 2_{[3 \overline{1} 0]}, \overline{3}_{[001]}^{+}, m_{(1 \overline{1} 0)}\right\} \\
& \cup\left\{6_{[001]}^{+}, 2_{[1 \overline{1} 0]}, \overline{6}_{[001]}^{+}, m_{(1 \overline{3} 0)}\right\} .
\end{aligned}
$$

Class $b$ :

$$
\begin{aligned}
6 / m m m= & \left\{1,2_{[100]}, \overline{1}_{,} m_{(100)}\right\} \cup\left\{3_{[001]}^{-}, 2_{[110]}, \overline{3}_{[001]}^{-}, m_{(130)}\right\} \\
& \cup\left\{6_{[001]}^{-}, 2_{[310]}, \overline{6}_{[001]}^{-}, m_{(110)}\right\} \\
& \cup\left\{2_{[001]}, 2_{[010]}, m_{(001)}, m_{(010)}\right\} \\
& \cup\left\{6_{[001]}^{+}, 2_{[3 \overline{1} 0]}, \overline{6}_{[001]}^{+}, m_{(1 \overline{1} 0)}\right\} \\
& \cup\left\{3_{[001]}^{+}, 2_{[1 \overline{1} 0]}, \overline{3}_{[001]}^{+}, m_{(1 \overline{3} 0)}\right\} .
\end{aligned}
$$

The first coset consists of the symmetry elements of $2 / m$ point group and the other five give the possible twin laws. Because of the nonzero obliquity, the four operations in each coset corresponding to a twin law are not equivalent. The law of Mallard requires that twin elements are lattice planes or rows and that twin operations are crystallographic operations. Therefore, the rotations and rotoinversions about [001] are not possible twin operations because they slightly deviate from $n \times 60^{\circ}$ [equation (1) is only approximately obeyed]. Peacock \& Ferguson (1943), Donnay et al. (1964) and Rieder (1970) concluded that the twin elements are the twofold axes in the (001) plane. However, when the composition plane is normal to (001) (see examples in Goldschmidt, 1918; Hurlbut, 1956; Sunagawa, 1964), the twin operation is a twin plane coinciding with the composition plane (Table 1).

\section{2. $3 T$ polytype}

In the case of the $3 T$ polytype (space group $P 3_{1,2} 12$ ), the twin lattice and the crystal lattice coincide.

The coset decomposition gives four cosets (indexing in $C_{1}$ setting):

$$
\begin{aligned}
6 / m m m= & \left\{1,3_{[001]}^{+}, 3_{[001]}^{-}, 2_{[010]}, 2_{310}, 2_{3 \overline{1} 0}\right\} \\
& \cup\left\{2_{[100]}, 2_{[110]}, 2_{[110]}, 2_{[001]}, 6_{[001]}^{-}, 6_{[001]}^{+}\right\} \\
& \cup\left\{m_{(100)}, m_{(130)}, m_{(1 \overline{3} 0)}, m_{(001)}, \overline{6}_{[001]}^{-}, 6_{[001]}^{+}\right\} \\
& \cup\left\{m_{(010)}, m_{(110)}, m_{(1 \overline{1} 0)}, \hat{i}, \overline{3}_{[001]}^{+}+\overline{3}_{[001]}^{-}\right\} .
\end{aligned}
$$


The first coset contains the symmetry elements of the 312 point group and the others the twin elements. These correspond to the three twin laws: $6^{\prime} 2^{\prime} 2 ; \overline{6}^{\prime} m^{\prime} 2 ; \overline{3}^{\prime} 12 / m^{\prime}$. The complete twin contains four individuals and has symmetry $6^{\prime} / m^{\prime \prime} 2^{\prime} / m^{\prime \prime} 2 / m^{\prime \prime \prime}$.

The six symmetry elements in each of the three cosets corresponding to a twin law are symmetrically equivalent under the operations of the point group 312: the twin law that actually operated appears in the crystal morphology and is called representative operation of the coset (Nespolo \& Ferraris, 2000). The $6^{\prime} 2^{\prime} 2$ and $\overline{6}^{\prime} m^{\prime} 2$ twin laws correspond to class IIA, according to the Nespolo \& Ferraris (2000) definition: for them, the twin elements do not belong to the point group of the family structure. This situation corresponds to 'selective merohedry' (Nespolo, Ferraris \& Durovič, 1999), which restores all the lattice nodes corresponding to the nonfamily reflections, but not all the lattice nodes corresponding to the family reflections of the threefold family structure. The presence of twinning is thus recognizable from the geometry of the diffraction pattern, since it shows violations of the non-space-group absences of the family reflec-

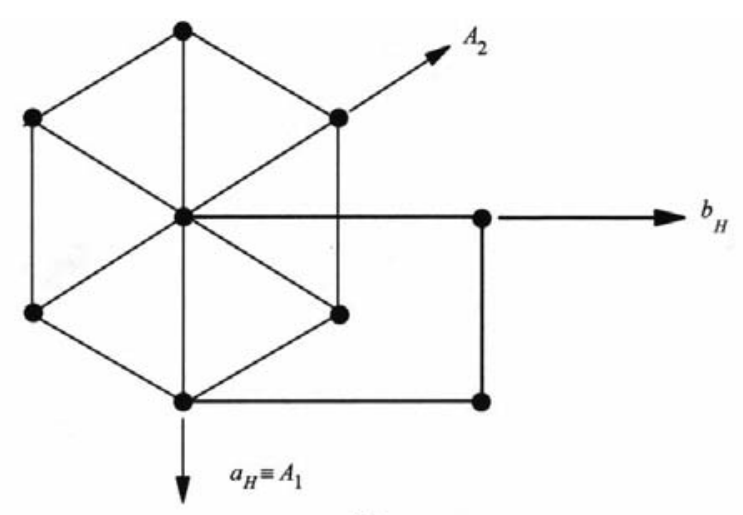

(a)

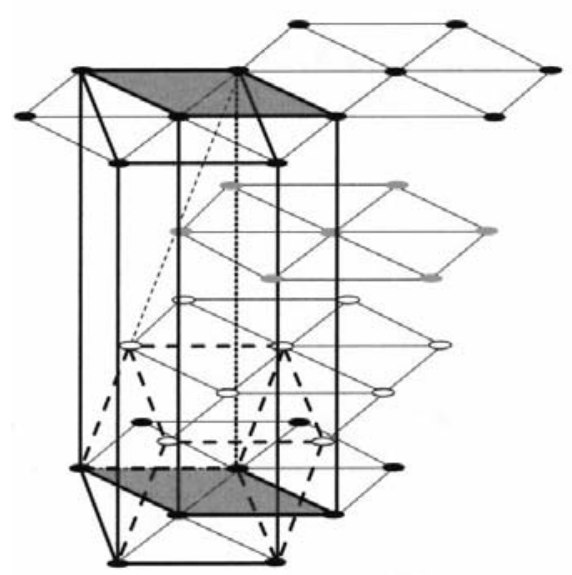

(b)

\section{Figure 3}

(a) Relation between hexagonal $\left(A_{1}, A_{2}\right)$ and orthohexagonal $\left(a_{H}, b_{H}\right)$ axes and location of the orthohexagonal cell $C_{1}$. $(b)$ The conventional monoclinic cell [(001) base dashed], the (pseudo)orthohexagonal cell (full lines), and the hexagonal cell (shaded) built overlapping three conventional cells. The scale along $\mathbf{c}$ is compressed (modified after Nespolo, Takeda \& Ferraris, 1997). tions. The $\overline{3^{\prime}} 12 / \mathrm{m}^{\prime}$ twin law corresponds to class I (Catti \& Ferraris, 1976; Nespolo \& Ferraris, 2000): all the twin elements belong to the point group of the family structure and twinning is by complete merohedry (Nespolo, Ferraris \& Durovič, 1999).

\subsection{Summary}

The twin elements for the four basic polytypes can be summarized as follows:

(i) $1 M$ and $2 M_{1}$ polytypes (Class a) undergo twinning by reticular pseudo-merohedry, with twin index 3 . There are five pairs of twin elements: [310]/(110), [31̄0]/(11̄0), [110]/(130), $[1 \overline{1} 0] /(1 \overline{3} 0)$ and $[100] /(100)$.

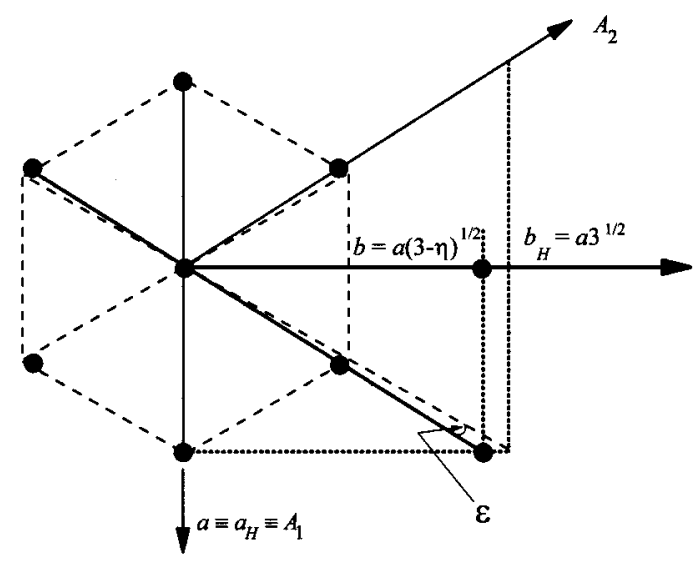

(a)

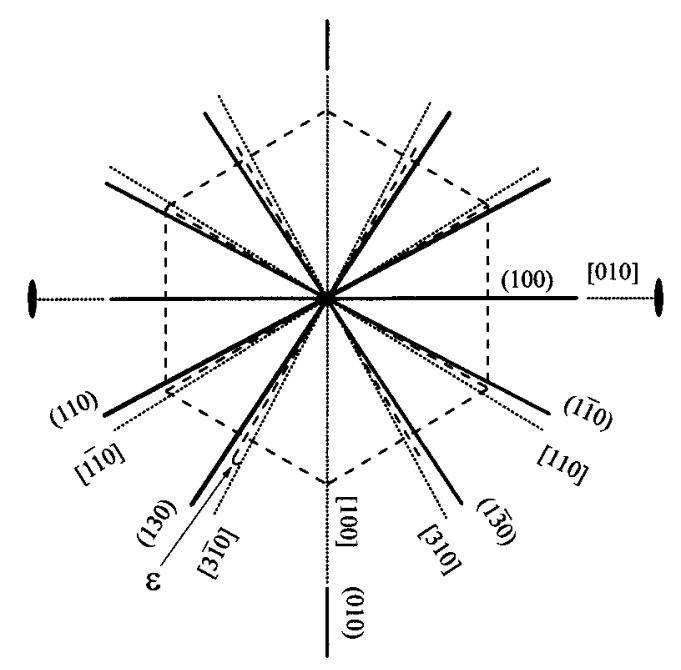

(b)

Figure 4

Projection onto (001) of the $C_{1}$ cell $(a)$ and location of the pseudosymmetry elements $(b) . A_{1}, A_{2}$ : hexagonal axes; $a_{H}, b_{H}$ : orthohexagonal axes; $a, b$ : pseudo-orthohexagonal axes. $\varepsilon$ and $\eta$ (exaggerated) are the angular and linear deviations from hexagonality. The figure is drawn for the case $b<b_{H}$. Black circles: lattice nodes of the crystal lattice; dashed lines: $H$ cell of the twin lattice; dotted lines: $C_{1}$ cell built on the hexagonal and pseudo-hexagonal meshes. $(b)$ Dashed lines: $H$ cell of the twin lattice; full lines: pseudo-symmetry planes; dotted lines: twofold axes quasinormal to the planes. [010] and (010) are symmetry elements for the conventional cell in the case of monoclinic Class a polytypes. For monoclinic Class $b$ polytypes, they become [100] and (100) (modified after Nespolo, Takeda \& Ferraris, 1997). 
Table 1

Twin laws for basic mica polytypes and corresponding relative rotations between pairs of twinned individuals.

\begin{tabular}{|c|c|c|c|c|c|c|c|c|c|c|c|c|c|}
\hline Polytype & $\begin{array}{l}\text { Point } \\
\text { group }\end{array}$ & Twinning & & \multicolumn{5}{|c|}{ Rotation twins } & \multicolumn{5}{|c|}{ Reflection twins } \\
\hline $3 T$ & 312 & $\begin{array}{l}\text { Complete } \\
\text { merohedry } \\
\text { Selective } \\
\text { merohedry }\end{array}$ & $\begin{array}{l}\text { Twin operation } \\
\text { Rotation } \\
\text { Twin operation } \\
\text { Rotation }\end{array}$ & $\begin{array}{l}{[100]_{\pi}} \\
(2 n+1) \times 60^{\circ}\end{array}$ & & & & & $\begin{array}{l}(010) \\
2 n \times 60^{\circ} \\
(100) \\
(2 n+1) \times 60^{\circ}\end{array}$ & & & & \\
\hline $1 M, 2 M_{1}$ & $2 / m$ & $\begin{array}{l}\text { Reticular } \\
\quad \text { pseudo-merohedry }\end{array}$ & $\begin{array}{l}\text { Twin operation } \\
\text { Rotation }\end{array}$ & $\begin{array}{l}{[310]_{\pi}} \\
240^{\circ}-2 \varepsilon\end{array}$ & $\begin{array}{l}{[110]_{\pi}} \\
300^{\circ}-2 \varepsilon\end{array}$ & $\begin{array}{l}{[3 \overline{1} 0]_{\pi}} \\
120^{\circ}+2 \varepsilon\end{array}$ & $\begin{array}{l}{[1 \overline{1} 0]_{\pi}} \\
60^{\circ}+2 \varepsilon\end{array}$ & $\begin{array}{l}{[100]_{\pi}} \\
180^{\circ}\end{array}$ & $\begin{array}{l}(110) \\
240^{\circ}+2 \varepsilon\end{array}$ & $\begin{array}{l}(130) \\
300^{\circ}+2 \varepsilon\end{array}$ & $\begin{array}{l}(1 \overline{1} 0) \\
120^{\circ}-2 \varepsilon\end{array}$ & $\begin{array}{l}(1 \overline{3} 0) \\
60^{\circ}-2 \varepsilon\end{array}$ & $\begin{array}{l}(100) \\
180^{\circ}\end{array}$ \\
\hline $2 M_{2}$ & $2 / m$ & $\begin{array}{l}\text { Reticular } \\
\quad \text { pseudo-merohedry }\end{array}$ & $\begin{array}{l}\text { Twin operation } \\
\text { Rotation }\end{array}$ & $\begin{array}{l}{[310]_{\pi}} \\
300^{\circ}-2 \varepsilon\end{array}$ & & $\begin{array}{l}{[3 \overline{1} 0]_{\pi}} \\
60^{\circ}+2 \varepsilon\end{array}$ & & $\begin{array}{l}{[010]_{\pi}} \\
180^{\circ}\end{array}$ & $\begin{array}{l}(110) \\
300^{\circ}+2 \varepsilon\end{array}$ & & $\begin{array}{l}(1 \overline{1} 0) \\
60^{\circ}-2 \varepsilon\end{array}$ & & $\begin{array}{l}(010) \\
180^{\circ}\end{array}$ \\
\hline
\end{tabular}

(ii) $2 M_{2}$ polytype (Class $b$ ) has five pairs of twin laws

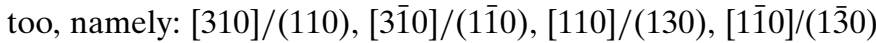
and $[010] /(010)$. Of these, the two pairs $[110] /(130)$ and $[1 \overline{1} 0] /(1 \overline{3} 0)$ are twin elements by pseudo-merohedry, whereas the remaining three pairs correspond to twinning by reticular pseudo-merohedry, with twin index 3.

(iii) $3 T$ polytypes have three twin laws, of which one $\left(\overline{3}^{\prime} 12 / m^{\prime}\right)$ corresponds to complete merohedry and the other two to selective merohedry $\left(6^{\prime} 2^{\prime} 2\right.$ and $\left.\overline{6}^{\prime} m^{\prime} 2\right)$.

The five pairs of twin elements of the monoclinic polytypes are mutually almost, but not exactly, perpendicular in the crystal lattice. The deviation from the perpendicularity can occur both in the (001) plane $(\varepsilon \neq 0$, Fig. $4 b)$ and in the $(h k 0)$ planes, which are not exactly perpendicular to the (001) plane (the $C_{1}$ cell is not exactly orthogonal). For the first four pairs of twin elements, common to both Classes, deviations in both planes occur. For the last pair, namely [100]/(100) for Class a and $[010] /(010)$ for Class $b$, the deviation in the (001) plane does not exist $(\varepsilon=0)$ but the second one in general remains. It follows that all the five pairs of twin laws can produce the socalled reciprocal twins (Mügge, 1898) or corresponding twins (Friedel, 1904, 1926). These are however hardly distinguishable from their diffraction pattern because of the small value of the twin obliquity.

\section{Symbolic description of the orientation of twinned mica individuals. Limiting symmetry}

Two twin laws are said to be equivalent when the corresponding twin elements are related by symmetry operations of the crystal. In accordance to the law of Mallard, a twin element is restricted to a lattice element, and the twin operation to a symmetry operation for the lattice (Santoro, 1974).

The three basic monoclinic polytypes all correspond to the point group $2 / \mathrm{m}$. Each of the rotational twin operations, premultiplied by a $180^{\circ}$ rotation about the monoclinic symmetry axis, is equivalent to a rotation about the normal to (001). If it is assumed that $\left|\mathbf{c}_{n}\right|=|a| / 3$ or $|b| / 3$, the $c$ axis of the $C_{1}$ cell is normal to (001). Only in the case of [100] (Class $a$ ) and [010] (Class $b$ ) does the angle between the twin element and the symmetry element $\left(90^{\circ}\right)$ not depend upon the twin obliquity, whereas for the other twin elements it corresponds to $n \times 30^{\circ} \pm \varepsilon$ (Fig. $4 b$ ). Two individuals twinned according to [100] (Class $a$ ) or [010] (Class $b$ ) are thus rotated by $180^{\circ}$ around the normal to the layer but the other four rotations correspond to $n \times 60^{\circ} \pm 2 \varepsilon$. For reflection twins, the same relative rotations are obtained through application of the (010) (Class $a$ ) or (100) (Class b) symmetry plane of the crystal but the deviations from $n \times 60^{\circ}$ rotation are in the opposite direction (Table 1 ). Since $\varepsilon$ is normally very small (Donnay et $a l ., 1964)$, the possible orientations of individuals in a twin are almost identical with the possible orientations of layers in a polytype, and the shortened $Z$ symbols used for polytypes (written as $Z_{T}, T$ indicating twin) can be applied also to twins. There are four main differences between $Z$ and $Z_{T}$ symbols:

(i) Since in a twin there cannot be two individuals oriented in the same way, the sequence of $Z_{T}$ never contains the same digit twice.

(ii) The $Z$ symbol of polytypes has to take into account the space group, whereas $Z_{T}$ deals only with the symmetry of the point group. The orthohexagonal setting of the first individual is taken to coincide with that of the twin lattice: the first individual is always fixed in orientation $Z_{T}=3$ (Fig. $1 b$ ), and the orientations of the others are determined by the twin laws.

(iii) Rotation by $180^{\circ}$ of the whole twinned edifice around the $a$ axis of the space-fixed reference changes the $3 I J \ldots P Z_{T}$ symbols into $(6-P) \ldots(6-J)(6-I) 3$; since the order of the individuals in the twin does not influence the diffraction pattern, this sequence is equivalent to $3(6-I)(6-J) \ldots(6-P)$. The latter corresponds to inverting the direction of rotation of the individuals in the twin. Considering the effect on the lattice, the $3 I J \ldots P \rightarrow 3(6-I)(6-J) \ldots(6-P)$ transformation corresponds to reflecting the twin lattice across the (010) plane.

(iv) The point group of the $3 T$ polytype contains only proper motions. The orientation symbol of an individual produced by a twin operation corresponding to an improper motion is hereinafter distinguished by a small black circle (•) after the $Z_{T}$ symbol, which indicates a reflection across a symmetry plane for the lattice [e.g. (010)].

The number of independent orientations of the reciprocal lattice of an individual is determined by the lower symmetry between the crystal lattice and the family structure, which hereinafter is called limiting symmetry. For Class a polytypes, the lattice symmetry is monoclinic and the family structure is 
Table 2

Orientation of the individuals building a twin in Class a mica polytype.

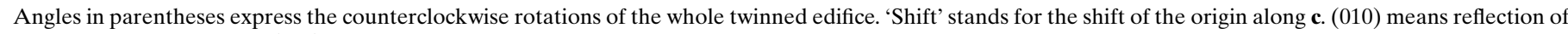

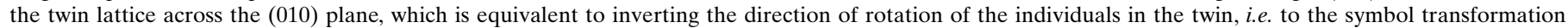
$3 I J \ldots P \rightarrow 3(6-I)(6-J) \ldots(6-P)($ see $\S 3)$.

\begin{tabular}{|c|c|c|c|c|c|c|}
\hline$Z_{T}$ & Equivalent to & Equivalent to & Equivalent to & $Z_{T}$ & Equivalent to & Equivalent to \\
\hline 34 & Unique & - & - & 3456 & Unique & - \\
\hline 35 & Unique & - & - & 3451 & Unique & - \\
\hline 36 & Unique & - & - & 3452 & $4563\left(60^{\circ}\right)$ & 3456 (shift) \\
\hline 31 & $53\left(120^{\circ}\right)$ & 35 (shift) & - & 3461 & Unique & - \\
\hline 32 & $43\left(60^{\circ}\right)$ & 34 (shift) & - & 3462 & Unique & - \\
\hline 345 & Unique & - & - & 3412 & $5634^{\prime}\left(120^{\circ}\right)$ & 3456 (shift) \\
\hline 346 & Unique & - & - & 3561 & $1345\left(240^{\circ}\right)$ & 3451 (shift) \\
\hline 341 & $325(010)$ & $436\left(60^{\circ}\right)$ & 346 (shift) & 3562 & $4613\left(60^{\circ}\right)$ & 3461 (shift) \\
\hline 342 & $453\left(60^{\circ}\right)$ & 345 (shift) & - & 3512 & $5134\left(120^{\circ}\right)$ & 3451 (shift) \\
\hline 356 & $134\left(240^{\circ}\right)$ & 341 (shift) & 346 & 3612 & $6345\left(180^{\circ}\right)$ & 3456 (shift) \\
\hline 351 & Unique & - & - & 34561 & Unique & - \\
\hline 352 & $463\left(60^{\circ}\right)$ & 346 (shift) & - & 34562 & $45613\left(60^{\circ}\right)$ & 34561 (shift) \\
\hline 361 & $634\left(180^{\circ}\right)$ & 346 & - & 34512 & $56134\left(120^{\circ}\right)$ & 34561 (shift) \\
\hline 362 & $413\left(60^{\circ}\right)$ & 341 (shift) & 346 & 34612 & $61345\left(180^{\circ}\right)$ & 34561 (shift) \\
\hline \multirow[t]{2}{*}{312} & $534\left(120^{\circ}\right)$ & 345 (shift) & - & 35612 & $13456\left(240^{\circ}\right)$ & 34561 (shift) \\
\hline & & & & 345612 & Unique & - \\
\hline
\end{tabular}

rhombohedral: the limiting symmetry is thus monoclinic. Besides, since the unique axes do not coincide ( $b$ in the polytypes, $c$ in the family structure), all the six orientations of the individual reciprocal lattice about the normal to (001) are independent. For a $2 M_{2}$ (Class $b$ ) polytype, the lattice symmetry is (pseudo)rhombohedral and the family structure is hexagonal: the limiting symmetry is thus rhombohedral: only two orientations of the individual reciprocal lattice are independent, corresponding to the two parities of $Z_{T}$ symbols. A common symbol is used for the three equivalent orientations with the same parity, namely $U$ (uneven) and $E$ (even). Finally, for the $3 T$ polytype, the crystal lattice is hexagonal and the family structure is rhombohedral: the limiting symmetry is rhombohedral and the two independent orientations of the individual w.r.l. are again $Z_{T}=U$ and $Z_{T}=E$.

\subsection{Class a polytypes}

Since all the six possible orientations of the individuals correspond to independent orientations of the reciprocal lattice, the possible composite twins can be obtained by calculating the sequences of $Z_{T}$ symbols for sets of individuals from two to six. The orientation of the first individual is fixed $\left(Z_{T}=3\right)$ and five possible orientations remain on which $m$ individuals $(1 \leq m \leq 5)$ have to be distributed. The number of twins is then:

$$
N_{T}(m)=\left(\begin{array}{c}
5 \\
m
\end{array}\right)=\frac{5 !}{m !(5-m) !} .
$$

Table 2 gives the corresponding 31 sequences of $Z_{T}$ symbols; only 12 of them represent independent twins. The other 19 simply correspond to a rotation of the whole twinned edifice followed by a shift of the origin along $\mathbf{c}$, eventually coupled with the inversion of the direction of the rotation of the individuals in the twin [reflection of the lattice across (010)], as in the case of $Z_{T}=341$.

\section{2. $2 M_{2}$ polytype}

Twinning by pseudo-merohedry involves individuals with the same orientation parity of $Z_{T}$ symbols and produces complete overlap of the individual reciprocal lattice. The reciprocal lattice of the twin is thus geometrically indistinguishable from the reciprocal lattice of the individual. The three twins $Z_{T}=35, Z_{T}=31$ and $Z_{T}=351$ are all equivalent to the single crystal, when considering the geometry of their lattice, and are thus represented as $Z_{T}=U$. Instead, twinning by reticular pseudo-merohedry involves individuals with opposite orientation parity of the $Z_{T}$ symbols and, considering the lattice only, they are all represented as $Z_{T}=U E$.

\section{3. $3 T$ polytype}

If the first individual is fixed in orientation $Z_{T}=U$, the effect of the three twin laws on it is:

$6^{\prime} 2^{\prime} 2$, twin operation $[100]_{\pi}$ or equivalent: $Z_{T}=U \rightarrow$ $Z_{T}=E$

$\overline{6}^{\prime} m^{\prime} 2$, twin operation (100) or equivalent, which can be expressed as (010) $\cdot[100]_{\pi}: Z_{T}=U \rightarrow Z_{T}=E^{\bullet}$;

$\overline{3}^{\prime} 12 / m^{\prime}$, twin operation (010) or equivalent: $Z_{T}=U \rightarrow$ $Z_{T}=U^{\bullet}$.

The first two twin operations correspond to selective merohedry (\$2.2). Because of the non-space-group absences of the family structure (see above), along each family row with $h \neq 0(\bmod 3)$ and $k=0(\bmod 3)$, two (one from each individual) out of three reflections appear, the third (ideally absent) always corresponding to $l=0(\bmod 3)$. The third twin operation corresponds to complete merohedry.

\section{Translationally independent reciprocal-lattice rows}

Since the $\mathbf{c}_{n}$ projection for non-orthogonal polytypes can ideally take only one of the two values $|a| / 3$ (Class $a$ ) or $|b| / 3($ Class $b)$, in the $C_{1}$ setting the metric relation in 
reciprocal space is $l_{C_{1}}(\bmod 3)=h(\bmod 3)($ Class $a)$ or $l_{C_{1}}(\bmod 3)=k(\bmod 3) \quad($ Class $b)$. By considering the $l_{C_{1}}(\bmod 3)$ index of reciprocal-lattice nodes on rows related by $n \times 60^{\circ}$ rotations $(0 \leq n \leq 5)$, it has been shown (Nespolo, Takeda, Ferraris \& Kogure, 1997) that in the case of the $1 M$ polytype there are only nine translationally independent rows parallel to $\mathbf{c}^{*}$ (Fig. 5). For the other polytypes, the violation of the additional reflection conditions in principle may cancel the geometrical equivalence between reciprocal-lattice rows. However, the forbidden reflections are weak enough to be neglected and the same classification developed for the $1 \mathrm{M}$ polytype holds thus in general (Nespolo, 1999b).

The translationally independent rows are hereinafter indicated as $R_{i}, 1 \leq i \leq 9$. In each $R_{i}$, the same distribution of 'present' and 'absent' reflections is repeated along $\mathbf{a}^{*}$ and $\mathbf{b}^{*}$ with $3 p$ and $3 q$ translations ( $p$ and $q$ are integers of the same parity). $R_{i}$ are defined in terms of $h$ and $k$ as: $\left[h_{i}(\bmod 3)\right.$, $\left.k_{i}(\bmod 3), l\right]$ and are distributed along the edges and diagonals of a rhombus-shaped unit, called a tessellation rhombus (Fig. 5, solid lines), which can tessellate the whole reciprocal space by $(3 p, 3 q)$ translations. A smaller unit, called minimal rhombus, can be drawn (Fig. 5, dotted lines), defined by the same $R_{i}$ each taken only once. Opposite edges are different and, contrary to the tessellation rhombus, the minimal rhombus does not represent a translational unit.

The two rhombi have six possible orientations (Fig. 5), which however represent equivalent descriptions of the same reciprocal lattice: they just differ in the distribution of the $R_{i}$. Six equivalent rhombi are obtained by applying all the five rotations (besides the identity) to the $h_{i}, k_{i}$ indices of each of

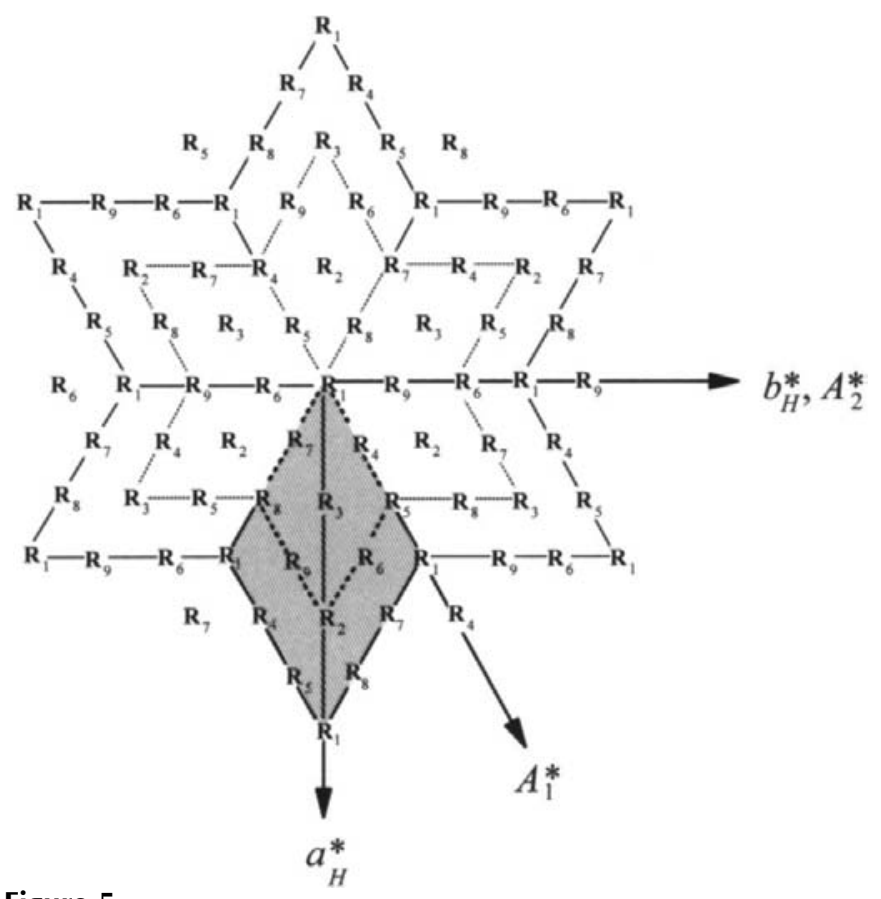

Figure 5

Minimal rhombus (dotted lines; in the foreground) and tessellation rhombus (solid lines) in the six orientations defining the star polygon. The nine translationally independent rows are distinguished by sequence numbers $\left(R_{1}-R_{9}\right)$. $A_{1}^{*}, A_{2}^{*}$ : hexagonal axes; $a_{H}^{*}, b_{H}^{*}$ : orthohexagonal axes (modified after Nespolo, Takeda, Ferraris \& Kagure, 1997). the nine $R_{i}$ of the original rhombus and bringing the resulting $R_{i}$ within the rhombus through a $(3 p, 3 q)$ translation between equivalent rows. The rows that can be reached by rotating the original rhombus are within a star-polygon located by the six rhombi with a common origin (Fig. 5). The values of $p$ and $q$ to be taken into account are those connecting rows internal to the star-polygon but external to the original rhombus with rows internal to the original rhombus, i.e. $(0, \pm 2),(1, \pm 1)$ and $(2, \pm 2)$.

\section{Classification of reciprocal-lattice rows}

In the $c_{1}^{*}$ repeat of nonfamily rows, as many reflections appear as the number $N$ of layers (some of which are very weak because of the additional reflection conditions), each separated by $c_{1}^{*} / N$. In the $C_{1}$ setting, the period along $\mathbf{c}^{*}$ is $c_{1}^{*} / N^{\prime}$ [see equation (2)]. The shortest repeat common to all the four basic polytypes is $c_{1}^{*} / 6$, which coincides with the period of $2 M_{1}$ and $2 M_{2}$ polytypes, and is a subperiod for $1 M$ and $3 T$ polytypes. By introducing a (pseudo)orthohexagonal setting with period $c_{1}^{*} / 6$ along $\mathbf{c}^{*}$, the $l$ indices of the nodes within a $c_{1}^{*}$ repeat can be indexed in a common cell for the four basic polytypes. This setting is here called 'twin setting', and the corresponding $l$ indices are indicated as $l_{T}: l_{T}\left(2 M_{1}, 2 M_{2}\right)=l_{C_{1}}\left(2 M_{1}, 2 M_{2}\right)$, but $l_{T}(1 M, 3 T)=$ $2 l_{C_{1}}(1 M, 3 T)$. The twin setting of the twin lattice is space-fixed and parallel to $C_{1}$, whereas that of the crystal lattice is crystalfixed for each of the individuals building a twin. Since the first individual of the twin is space-fixed $\left(Z_{T}=3\right.$ for Class $a$ or $Z_{T}=U$ for $2 M_{2}$ and $3 T$ ), its twin setting is parallel to $C_{1}$.

Hereinafter, the 'row features' characterize the reciprocallattice rows parallel to $\mathbf{c}^{*}$ as a whole and the 'node features' characterize each single reciprocal-lattice row, expressing the number and disposition of reciprocal-lattice nodes on them. If the deviation from orthohexagonality $(\varepsilon, \eta)$ is neglected, all mica polytypes share the same row features, described by the regular tessellation $\{3,6\}$ (Takeda \& Donnay, 1965) and the nine $R_{i}$ can be classified into three types (Fig. 6):

(i) $S$ (single) rows $[h=0(\bmod 3)$ and $k=0(\bmod 3)]$. They are family rows of the ninefold structure (Pauling model): the single reflection always corresponds to $l_{T}=0(\bmod 6)$.

(ii) $D$ (double) rows $[h \neq 0(\bmod 3)$ and $k=0(\bmod 3)]$. They are family rows of the threefold structure (trigonal model), but nonfamily rows for mixed-rotation polytypes. There are two independent $D$ rows, labelled $D_{i}: i=1,2$; $h=i(\bmod 3) ; k=0(\bmod 3)$.

(iii) $X$ (sextuple) rows $[k \neq 0(\bmod 3)]$. They are nonfamily rows for all polytypes. There are six independent $X$ rows, labelled $X_{i}: 1 \leq i \leq 6 ; h=i(\bmod 3) ; k=2 \times(-1)^{i}(\bmod 3)$.

The nine $R_{i}$ rows are thus classified as: $R_{1}=S ; R_{2-3}=D_{1-2}$; $R_{4-9}=X_{1-6} \cdot{ }^{\mathbf{6}}$ Each of the three types of rows lies on non-

\footnotetext{
${ }^{6}$ Sadanaga \& Takéuchi (1961), dealing with polysynthetic twins of $1 M$, classified the reciprocal-lattice rows into $S$ rows $(S=\operatorname{single})$, twin rows and $T$ rows $(T=$ triple $)$, recalling the number of reflections in a $c_{1}^{*}$ repeat. That terminology is meaningful when dealing with $1 M$ twins, but not in a general approach. The present classification is a general feature of the $\{3,6\}$ regular tessellation and does not depend on the polytype.
} 
Table 3

Definition of the $I_{j}$ for the four basic polytypes and their twins.

$I$ indicates the number of nodes on the reciprocal-lattice row. The subscript $j$ is a sequential number (see $§ 5$ ).

\begin{tabular}{|c|c|c|c|c|c|c|c|c|c|c|c|c|c|c|c|c|}
\hline $\begin{array}{l}I_{j} \\
l(\bmod 6) \\
I_{j} \\
l(\bmod 6)\end{array}$ & $\begin{array}{l}1_{1} \\
0 \\
4_{1} \\
0,1,2,4\end{array}$ & $\begin{array}{l}1_{2} \\
2 \\
4_{2} \\
0,1,3,4\end{array}$ & $\begin{array}{l}1_{3} \\
4 \\
4_{3} \\
0,2,3,5\end{array}$ & $\begin{array}{l}2_{1} \\
0,2 \\
4_{4} \\
0,2,3,4\end{array}$ & $\begin{array}{l}2, \\
0,4 \\
4_{5} \\
0,2,4,5\end{array}$ & $\begin{array}{l}2, \\
0,3 \\
4_{6} \\
1,2,4,5\end{array}$ & $\begin{array}{l}2_{4} \\
1,4 \\
5_{1} \\
0,1,2,3,4\end{array}$ & $\begin{array}{l}2_{5} \\
2,5 \\
5_{2} \\
0,1,2,4,5\end{array}$ & $\begin{array}{l}2_{6} \\
2,4 \\
5_{3} \\
0,2,3,4,5\end{array}$ & $\begin{array}{l}3_{1} \\
0,2,4 \\
6_{1} \\
0,1,2,3,4,5\end{array}$ & $\begin{array}{l}3_{2} \\
0,1,4\end{array}$ & $\begin{array}{l}3_{3} \\
0,2,3\end{array}$ & $\begin{array}{l}3_{4} \\
0,2,5\end{array}$ & $\begin{array}{l}3_{5} \\
0,3,4\end{array}$ & $\begin{array}{l}3_{6} \\
1,2,4\end{array}$ & $\begin{array}{l}3_{7} \\
2,4,5\end{array}$ \\
\hline
\end{tabular}

intersecting circular orbits centred on $c^{*}$. An $n \times 60^{\circ}$ rotation overlaps rows belonging to the same type only. In Fig. 6, heavy and thin circles describe the orbits of family ( $S$ full lines and $D$ dashed lines) and nonfamily $(X)$ rows, respectively. For $X$ rows, two kinds of orbits exist, connecting one (full lines) and two (dashed lines) sets of six rows. The $n \times 60^{\circ}$ rotations about c $^{*}$ lead to an alternate exchange of the two $D$-type $R_{i}$ located on the long diagonal of the minimal rhombus, and they exchange the six $X$-type $R_{i}$ on the edges of the minimal rhombus in six different ways.

The transformations for the $h_{i}, k_{i}$ indices of the nine $R_{i}$ in the six orientations of the minimal rhombus are shown in Fig. 7, where the distribution of the $X_{i}$ is given by the arrows. To each of these six orientations, the reflection across the (010) plane, which corresponds to the $Z_{T}=3 I J \ldots P \rightarrow$ $3(6-I)(6-J) \ldots(6-P)$ transformation (see $\S 3)$, has to be applied. The second set is obtained from the first one through the sequence $X_{4} \rightarrow X_{5} \rightarrow X_{6} \rightarrow X_{1} \rightarrow X_{2} \rightarrow X_{3}$ applied to

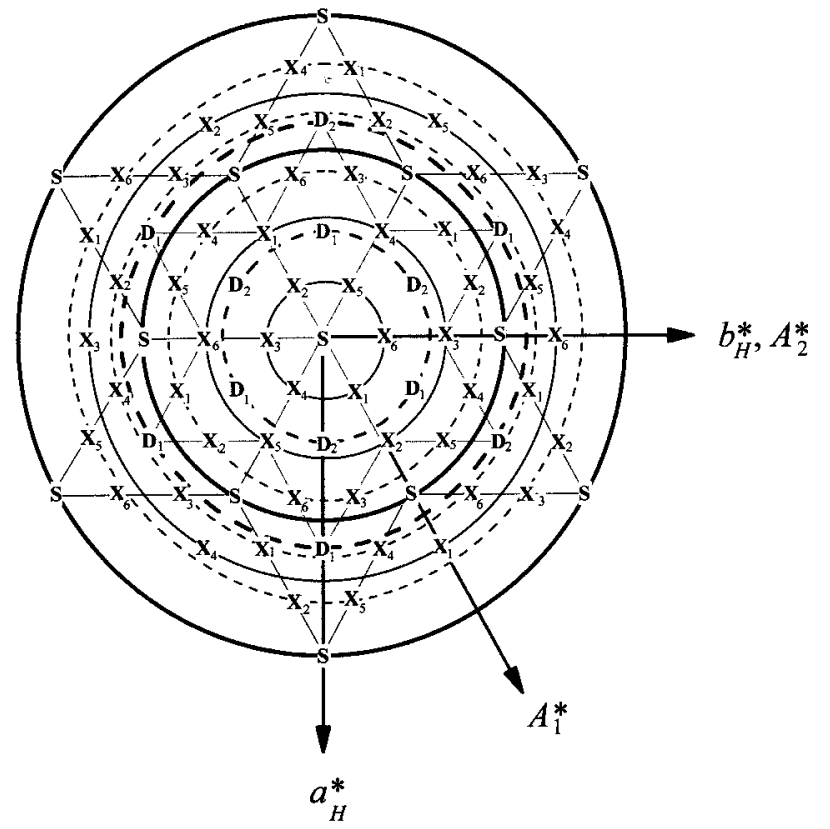

Figure 6

Rotational relation between reciprocal-lattice rows parallel to $\mathbf{c}^{*}$ and definition of the rotational sequences $\left(\mathrm{RS}_{i}^{P}\right) . A_{1}^{*}, A_{2}^{*}$ : hexagonal axes; $a_{H}^{*}, b_{H}^{*}$ : orthohexagonal axes. Because of the pseudo-hexagonal symmetry of the (001) plane, each type of row $(S, D, X)$ lies on a circular orbit around $\mathbf{c}^{*}$. Heavy orbits: family rows (full lines: $S$ rows; dashed lines: $D$ rows); thin orbits: nonfamily $(X)$ rows (full lines: one set of six rows; dashed lines: two sets of rows). The $n \times 60^{\circ}$ rotations, which correspond to the relative orientation of twinned or allotwinned mica individuals, do not mix different types of row $(S, D$ and $X)$. the original $\left(0^{\circ}\right)$ orientation. Two minimal rhombi that can be transformed into each other by application of one of these 12 operations are equivalent.

The node features (number and disposition of nodes on the reciprocal-lattice rows) are hereinafter identified by a symbol $I_{j}$, where $I$ is the number of nodes within the $c_{1}^{*}$ repeat and $j$ is a sequence number. The definition of $I_{j}$ is given in Table 3 . Seven $I_{j}$ are distributed on the nine $R_{i}$ of the four basic polytypes (Table 4): $26 I_{j}$ are obtained by multiplying these seven $I_{j}$ : they form a group, as shown in the multiplication table (Table 5).

\section{Derivation of twin diffraction patterns}

The reciprocal-lattice rows of a twin derive from the overlap of single-crystal rows $\left(R_{i}\right)$ and are called 'composite rows'

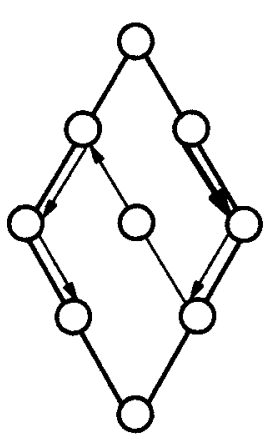

$0^{\circ}$

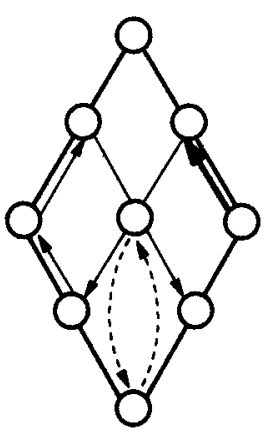

$180^{\circ}$

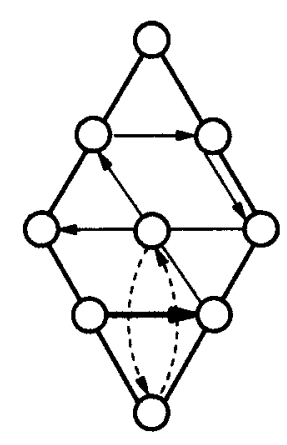

$60^{\circ}$

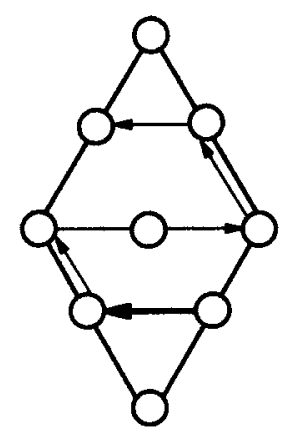

$240^{\circ}$

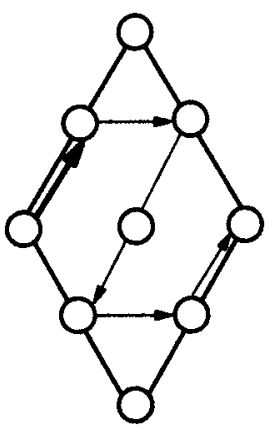

$120^{\circ}$

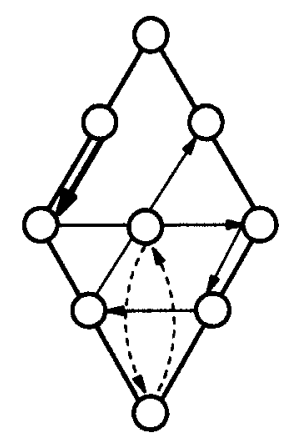

$300^{\circ}$
Figure 7

Distribution of the six $X$ rows along the edges of the minimal rhombus as a function of the rotation of the minimal rhombus itself. The arrows indicate the reading sequence corresponding to the rotation angle given below the rhombus. The two $D$ rows are unchanged for $\pm 120^{\circ}$ but exchanged for \pm 60 or $180^{\circ}$ rotations. 


\section{Table 4}

$R_{i}$ for the four basic polytypes (cf. Figs. 5 and 6).

For the definition of $I_{j}$, see Table 3.

\begin{tabular}{|c|c|c|c|c|c|c|c|c|c|c|c|}
\hline \multirow[b]{2}{*}{ Single-crystal row } & \multirow[b]{2}{*}{ Type } & \multirow[b]{2}{*}{$h(\bmod 3)$} & \multirow[b]{2}{*}{$k(\bmod 3)$} & \multicolumn{2}{|l|}{$1 M$} & \multicolumn{2}{|l|}{$2 M_{1}$} & \multicolumn{2}{|l|}{$2 M_{2}$} & \multicolumn{2}{|l|}{$3 T$} \\
\hline & & & & $l(\bmod 6)$ & $I_{j}$ & $l(\bmod 6)$ & $I_{j}$ & $l(\bmod 6)$ & $I_{j}$ & $l(\bmod 6)$ & $I_{j}$ \\
\hline$R_{1}$ & $S$ & 0 & 0 & 0 & $1_{1}$ & 0 & $1_{1}$ & 0 & $1_{1}$ & 0 & $1_{1}$ \\
\hline$R_{4}$ & $X_{1}$ & 1 & 1 & 2 & $1_{2}$ & 1,4 & $2_{4}$ & 1,4 & $2_{4}$ & $0,2,4$ & $3_{1}$ \\
\hline$R_{5}$ & $X_{2}$ & 2 & 2 & 4 & $1_{3}$ & 2,5 & 25 & 2,5 & 25 & $0,2,4$ & $3_{1}$ \\
\hline$R_{6}$ & $X_{3}$ & 0 & 1 & 0 & $1_{1}$ & 0,3 & 23 & 1,4 & 24 & $0,2,4$ & $3_{1}$ \\
\hline$R_{9}^{\circ}$ & $X_{6}$ & 0 & 2 & 0 & $1_{1}$ & 0,3 & $2{ }_{3}$ & 2,5 & 25 & $0,2,4$ & $3_{1}$ \\
\hline
\end{tabular}

\section{Table 5}

Multiplication table for composite rows.

An $I_{j}$ in the body of the table is the product of the corresponding two $I_{j}$ in the first rows $\left(I_{j}^{A}\right)$ and in the first column $\left(I_{j}^{B}\right)$. For the definition of $I_{j}$, see Table 3 .

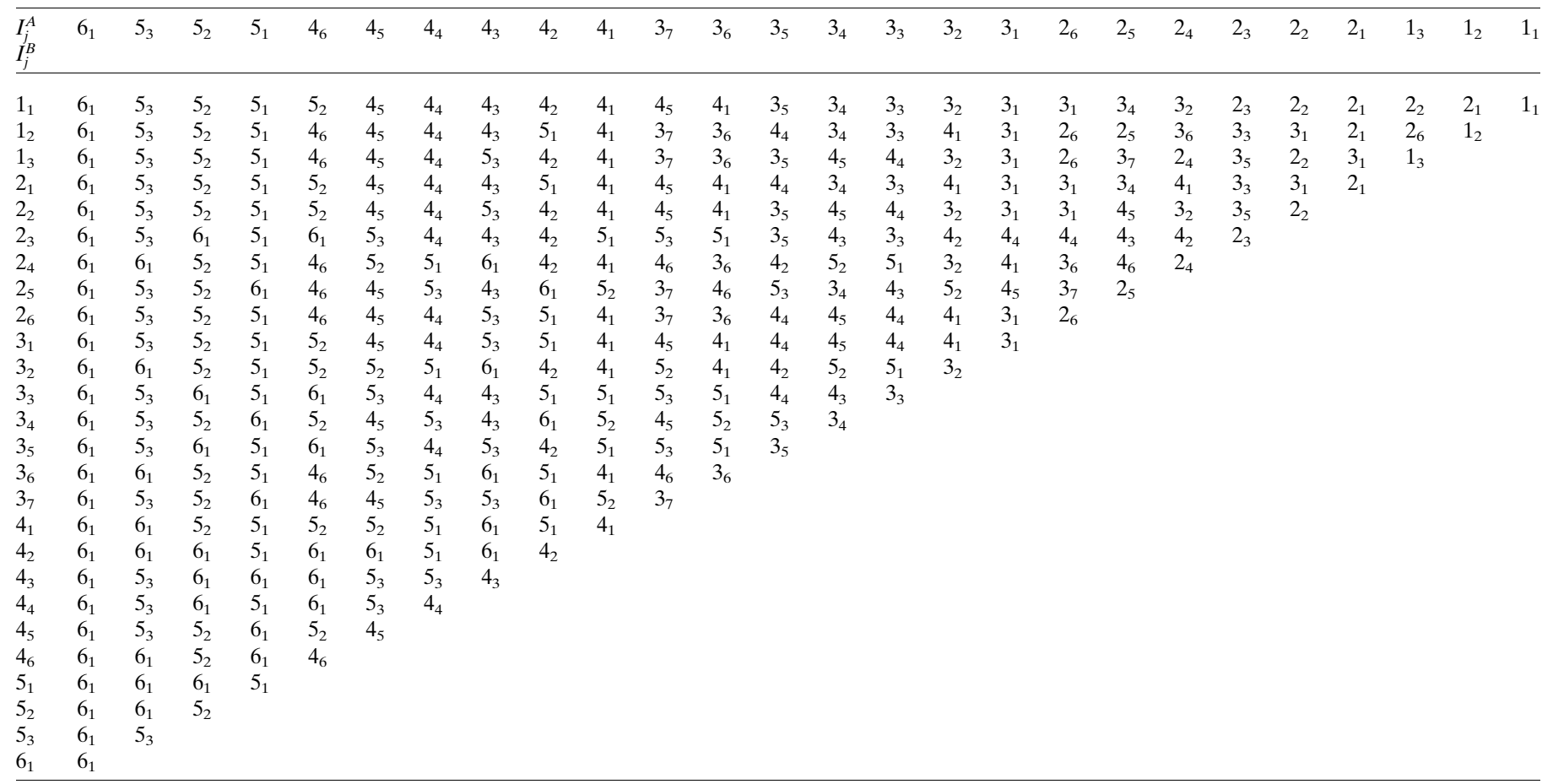

(Sadanaga \& Takéuchi, 1961). The analysis of $R_{i}$ represents the starting point in the derivation of twin patterns.

The $n \times 60^{\circ}$ rotations about $\mathbf{c}^{*}$ overlap only $R_{i}$ belonging to the same type $(S, D$ or $X)$, but specific nodes may overlap or not depending upon which $I_{j}$ corresponds to the rotationally related $R_{i}$. Each of the $R_{i}$ is rotationally related to five other ones and along each of them a peculiar sequence of $l_{T}$ indices is obtained, which has been called 'rotational sequence' (Nespolo, Takeda, Ferraris \& Kogure, 1997). Each $R_{i}$ generates one rotational sequence, which is shortened to $\operatorname{RS}_{i}^{P}(n)$, where the superscript $P$ indicates the polytype $\left(P=1 M, 2 M_{1}\right.$, $\left.2 M_{2}, 3 T\right) ; i$ is the same index defining $R_{i} ; n$ points to each of the six digits of the RS. $\mathrm{RS}_{1}^{P}$ always corresponds to '000000' for all polytypes. The $n$th values of $\mathrm{RS}_{1}^{P}$ correspond to the $l_{T}$ indices of the nodes on the row which is related to $R_{i}$ by $(n-1) \times 60^{\circ}$ clockwise rotation. The two $\mathrm{RS}_{1}^{P}$ corresponding to $D$-type rows $\left(R_{2-3}\right)$ on the one hand and the six $\mathrm{RS}_{1}^{P}$ corresponding to $X$-type rows $\left(R_{4-9}\right)$ on the other can be transformed into each other by cyclic permutations. Since the orientations of the single-crystal lattices and of the twin lattice are fixed and determined by $Z_{T}$, also the starting point of each $\mathrm{RS}_{1}^{P}$ is fixed, and the nine $\mathrm{RS}_{1}^{P}$ are independent. The node features of the composite rows can be obtained from the corresponding $\mathrm{RS}_{1}^{P}$ by considering their relation with the $Z_{T}$ symbols. A twin of $N$ individuals $(2 \leq N \leq 6)$ is identified by $N$ $Z_{T}$ symbols. The $l_{T}$ index of the $q$ th node on the $i$ th row from the $j$ th individual is given by:

$$
\left[l_{T}(i, j)\right]_{q}=\left[\operatorname{RS}_{i}^{P}(n)\right]_{q}, \quad n=\left[\left(Z_{T}\right)_{j}+4\right](\bmod 6) .
$$


Table 6

Composite rows $\left(C_{i}\right)$ and rotational sequences $\left(\operatorname{RS}_{i}^{P}\right)$ for the four basic polytypes (see $\S 6$ and Figs. 5 and 6).

\begin{tabular}{llllllll}
\hline Composite row & Type & $h(\bmod 3)$ & $k(\bmod 3)$ & $\mathrm{RS}^{1 M}$ & $\mathrm{RS}^{2 M_{1}}$ & $\mathrm{RS}^{2 M_{2}}$ \\
\hline$C_{1}$ & $S$ & 0 & 0 & 000000 & 000000 & 000000 & $\mathrm{RS}^{3 T}$ \\
$C_{2}$ & $D_{1}$ & 1 & 0 & 242424 & 424242 & $000000 / 333333$ & 242424 \\
$C_{3}$ & $D_{2}$ & 2 & 0 & 424242 & 242424 & $000000 / 333333$ \\
$C_{4}$ & $X_{1}$ & 1 & 1 & 204402 & $102201 / 435544$ & $121212 / 454545$ & $00000000 / 222222 / 444444$ \\
$C_{5}$ & $X_{2}$ & 2 & 2 & 402204 & $201102 / 534435$ & $212121 / 545454$ & $000000 / 222222 / 444444$ \\
$C_{6}$ & $X_{3}$ & 0 & 1 & 022044 & $011022 / 344355$ & $121212 / 454545$ & $000000 / 222222 / 444444$ \\
$C_{7}$ & $X_{4}$ & 1 & 2 & 220440 & $110220 / 443553$ & $212121 / 545454$ & $000000 / 222222 / 444444$ \\
$C_{8}$ & $X_{5}$ & 2 & 1 & 440220 & $220110 / 553443$ & $121212 / 454545$ & $000000 / 222222 / 444444$ \\
$C_{9}$ & $X_{6}$ & 0 & 2 & 044022 & $022011 / 355344$ & $212121 / 545454$ & $000000 / 222222 / 444444$ \\
\hline
\end{tabular}

The node features of composite rows are completely defined by the nine $\mathrm{RS}_{1}^{P}$ and $Z_{T}$ symbols; therefore, there are only nine independent composite rows, for which the symbol $C_{i}$ is adopted (Table 6). $R_{i}$ and $C_{i}$ share the same row features and thus the description of the reciprocal lattice in terms of the tessellation rhombus and of the minimal rhombus is the same for both the single-crystal lattice and the twin lattice.

Because of the metric relations ( $\$ 1.2)$, the $I_{j}$ of both $C_{i}$ and $R_{i}$ of the same type and belonging to the same central plane are related by:

$$
\begin{aligned}
& {\left[l_{T}\left(D_{i}\right)\right]_{q}=\left[6-l_{T}\left(D_{3-i}\right)\right]_{q}} \\
& {\left[l_{T}\left(X_{i}\right)\right]_{q}=\left\{6-l_{T}\left[X_{(9-i)(\bmod 6)}\right]\right\}_{q} .}
\end{aligned}
$$

With the $I_{j}$ of one $D$-type $C_{i} / R_{i}$ and three $X$-type $C_{i} / R_{i}$, the $I_{j}$ of the remaining four $C_{i} / R_{i}$ can be calculated. There are thus five truly geometrically independent $C_{i} / R_{i}$ (one $S$-type, one $D$-type and three $X$-type), but nine translationally independent $C_{i} / R_{i}$.

The distribution of $I_{j}$ on the $C_{i}$ of a minimal rhombus is the information necessary to derive and identify the diffraction patterns of mica twins.

\section{1. $1 M$ polytype}

The minimal rhombus analysis of $1 M$ twins given in Nespolo, Takeda, Ferraris \& Kogure (1997) is revised here in terms of $Z_{T}$ symbols.

The $c_{1}^{*}$ repeat coincides with the polytype period and along each $R_{i}$ there is only one node, which obeys the relation $l_{T}=2 h(\bmod 6) . D$-type $R_{i}$ are either $1_{2}\left(D_{1}\right)$ or $1_{3}\left(D_{2}\right)$ and the $\mathrm{RS}_{2-3}^{1 M}$ are 242424 and 424242 . The $n \times 60^{\circ}$ rotations about $\mathbf{c}^{*}$ produce the overlap of all the reciprocal-lattice nodes belonging to $D$-type $R_{i}$ when $n$ is even, but to their separation when $n$ is odd. $X$-type $R_{i}$ include all the three $1_{1}, 1_{2}$ and $1_{3}$ and the $\mathrm{RS}_{4-9}^{1 M}$ are the six cyclic permutations of 220440 . On the basis of the relation between $C_{i}$ and $\mathrm{RS}_{i}^{1 M}$ (Table 6) and of the multiplication table (Table 5), seven different $C_{i}$ appear in the twin lattice. Up to 2 reflections can appear on $D$-type $C_{i}$ $\left(I_{j}=1_{2}, 1_{3}\right.$ or $2_{6} ; l_{T}$ is never 0$)$, whereas up to three reflections (all the seven possible $I_{j}$ ) can appear on $X$-type $C_{i} . I_{j}$ define nine independent $1 M$ twin patterns (Fig. 8).

Nespolo, Takeda, Ferraris \& Kogure (1997) expressed the relative rotations between the first and the $(j+1)$ th individual as a sequence of $n_{j}\left(\bmod 60^{\circ}\right)$ integers. Those symbols are not space-fixed and in some respects are midway between the
$Z$ symbols and the rotational RTW symbols (Ross et al., 1966). The correspondence with $Z_{T}$ symbols is simply $Z_{T}(1)=3$; $Z_{T}(j+1)=3+n_{j}$. Consideration of the relative rotations, instead of absolute orientations, revealed ten patterns, of which however one (corresponding to $Z_{T}=341$ ) turns out to be not independent when the lattice is reflected across (010) [symbol transformation $Z_{T}=3 I J \ldots P \rightarrow$ $3(6-I)(6-J) \ldots(6-P)($ Table 2$)]$.

As seen above, when three equally spaced reflections in the $c_{1}^{*}$ repeat appear along nonfamily rows, in principle the
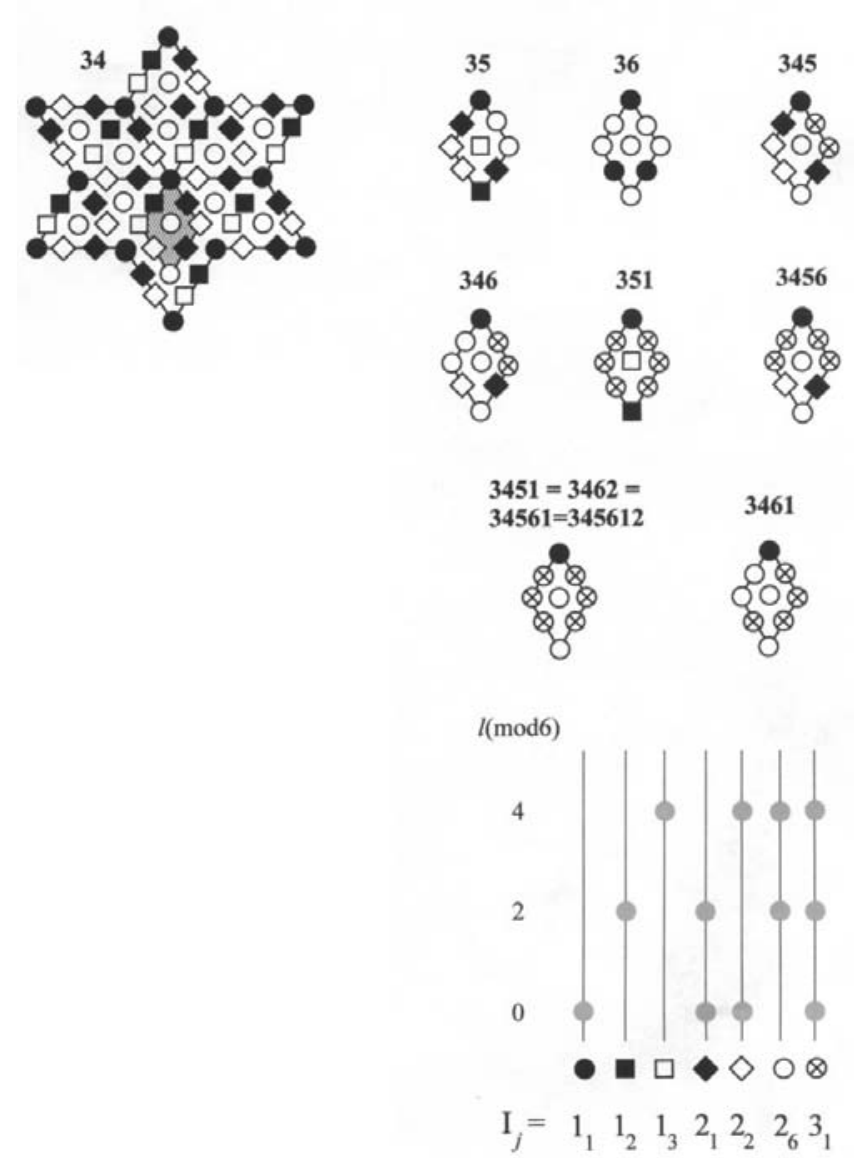

Figure 8

The nine independent patterns of $1 M$ twins as expressed through the corresponding minimal rhombi. For the $Z_{T}=34$ twin, the complete star polygon is given, with the minimal rhombus in it shaded. Inset on the lower right: $l(\bmod 6)$ indices of the nodes on reciprocal-lattice composite rows. 
diffraction pattern may correspond either to a $1 M$ twin (apparent polytypism) or to a three-layer polytype (real polytypism). The distinction is easily obtained by applying the geometrical criteria given in Nespolo (1999a). However, $1 M$ twins with $Z_{T}=351$ and the $3 T$ polytype $(Z=351$ or 315$)$ cannot be distinguished geometrically (see also Nespolo \& Kogure, 1998). In the case of dioctahedral and Li-Fe trioctahedral micas, this ambiguity is removed when weak reflections appear along family rows (Rieder, 1968, 1970) (see \$8).

\section{2. $2 M_{1}$ polytype}

Since the parity of layers is even $(Z=24)$ for a $2 M_{1}$ polytype but odd for a $1 M$ polytype, the threefold family structure has opposite settings (reverse/obverse) and the corresponding family rows have different reflection conditions, namely $k=0(\bmod 3), l_{T}=2 h(\bmod 6)$ for $1 M$, but $k=0(\bmod 3)$, $l_{T}=4 h(\bmod 6)$ for $2 M_{1}($ Nespolo, $1999 a)$. One reflection appears in the $c_{1}^{*}$ repeat along family $R_{i}$, but two along nonfamily $R_{i}$, with the following presence criteria:

$$
\left\{\begin{array}{lr}
k \neq 0(\bmod 3) & \left\{\begin{array}{l}
l_{T 1}=h(\bmod 6) \\
l_{T 2}=(h+3)(\bmod 6) \\
k=0(\bmod 3)
\end{array} \quad l_{T}=(h+k)(\bmod 6)\right.
\end{array}\right.
$$

The node features of $R_{i}$ are compared with those of the other basic polytypes in Table 4 . $D$-type $R_{i}$ are either $1_{2}$ or $1_{3}$, as in the $1 M$ case but, because of the opposite parity of the layers in the two polytypes, the positions of the two $I_{j}$ and the two $\mathrm{RS}_{2-3}^{2 M_{1}}$ are inverted. $X$-type $R_{i}$ have all the three possible pairs of values of $l_{T}(\bmod 6): 0$ and $3\left(I_{j}=2_{3}\right), 1$ and $4\left(I_{j}=2_{4}\right)$, 2 and $5\left(I_{j}=2_{5}\right)$. For the $X$-type $R_{i}$, the sequence of $n \times 60^{\circ}$ rotations corresponds to a double sequence of $l_{T}$ values: $011022 / 344355$ or cyclic permutations, producing six independent double $\mathrm{RS}_{i}^{2 M_{1}}$ (Table 6). As in the case of the $1 M$ polytype, the twelve composite twins produce nine different patterns, none of which can be mistaken for that of a $1 M$ twin (Fig. 9).

\section{3. $2 M_{2}$ polytype}

Being a Class $b$ polytype, $2 M_{2}$ has a markedly pseudorhombohedral lattice and two of the five pairs of twin laws, namely those corresponding to $\pm 120^{\circ}$ rotation about $\mathbf{c}^{*}$, correspond to pseudo-merohedry but the remaining three to reticular pseudo-merohedry. All the six $n \times 60^{\circ}$ rotations belong to the point group of the family structure (subfamily $B$ ), and thus the family sublattice of the individuals is always overlapped. The presence criteria in the twin setting are:

$$
\left\{\begin{array}{l}
k \neq 0(\bmod 3)\left\{\begin{array}{l}
l_{T 1}=k(\bmod 6) \\
l_{T 2}=(k+3)(\bmod 6)
\end{array}\right. \\
k=0(\bmod 3)\left\{\begin{array}{l}
h=0(\bmod 3): l_{T}=0(\bmod 6) \\
h \neq 0(\bmod 3)\left\{\begin{array}{l}
l_{T 1}=0(\bmod 6) \\
l_{T 2}=3(\bmod 6) .
\end{array}\right.
\end{array}\right.
\end{array}\right.
$$

$\mathrm{RS}_{2}^{2 M_{2}}$ and $\mathrm{RS}_{3}^{2 M_{2}}$ both correspond to the double sequence $000000 / 333333$, whereas the $\mathrm{RS}_{4-9}^{2 M_{2}}$ correspond to the cyclic permutations of the double sequence 121212/454545. There are only two kinds of patterns for $2 M_{2}$ twins. Twinning by pseudo-merohedry gives a pattern geometrically indistinguishable from that of the single crystal $\left(Z_{T}=U\right)$. The other pattern corresponds to twinning by reticular pseudomerohedry $\left(Z_{T}=U E\right)$ and differs from the single-crystal pattern in the six $X$-type $C_{i}$, which are all $I_{j}=4_{6}$ (see Tables 3 and 5). Neither of the two can be mistaken for any of the $1 M$ or $2 M_{1}$ polytypes or twins.

\section{4. $3 T$ polytype}

The $3 T$ polytype is an orthogonal subfamily $A$ polytype, with odd parity of the layers (the same as $1 M)$. $D$-type $R_{i}$ and $\mathrm{RS}_{i}^{3 T}$ are exactly the same as those of the $1 M$ polytype, but the $X$-type $R_{i}$ correspond to $I_{j}=3_{1}$ and there is thus only one triple sequence of $X$-type $\mathrm{RS}^{3 T}: 00000 / 222222 / 444444$. The six orientations of the minimal rhombus are divided into two types, differing for the $I_{j}$ corresponding to $D_{1}$ and $D_{2}$. The $2 n \times 60^{\circ}$ rotations belong to the point group of the individual and reproduce the same rhombus. On the other hand, $(2 n+1) \times 60^{\circ}$ rotations do not belong to the point group of the individual and thus they exchange the two independent rhombi.

Twinning by complete merohedry $\left(Z_{T}=U U^{\bullet}\right)$ by definition produces a diffraction pattern with the same geometrical appearance as the single crystal, which in its turn may be geometrically identical to the pattern of $1 M$ twinned as $Z_{T}=351$. On the contrary, in the case of twinning by selective merohedry $\left(Z_{T}=U E, U E^{\bullet}, U U^{\bullet} E, U U^{\bullet} E^{\bullet}, U U^{\bullet} E E^{\bullet}\right)$, the two $D$-type $C_{i}$ correspond to $2{ }_{6}$. This is the same geometrical appearance of $1 M$ twinned as $Z_{T}=3451$. The distinction between $1 M$ twins and the $3 T$ polytype (twinned or
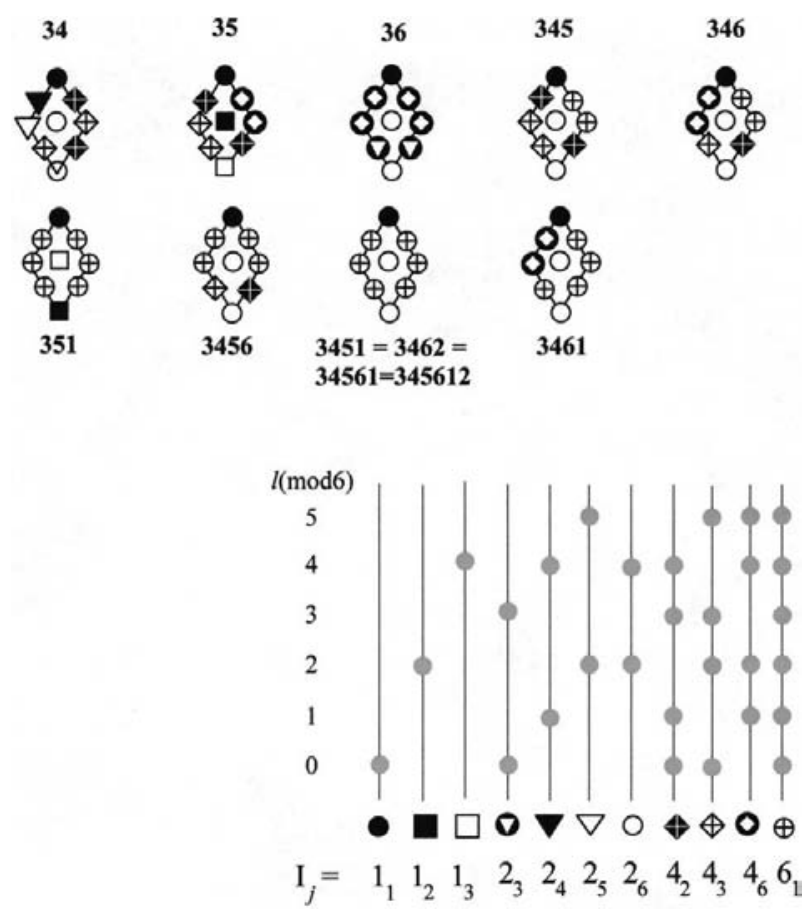

Figure 9

The nine independent patterns of $2 M_{1}$ twins as expressed through the corresponding minimal rhombi. Inset on the lower right: $l(\bmod 6)$ indices of the nodes on reciprocal-lattice composite rows. 
untwinned) has to be reached by very careful examination of the violation of the additional reflection conditions (see §8).

\section{Derivation of allotwin diffraction patterns}

The oriented association of two (or more) individuals differing only in the polytypic character is called allotwinning [from the Greek $\alpha \lambda \lambda \sigma_{5}$, 'different', with reference to the individuals (Nespolo, Kogure \& Ferraris, 1999)]. Since the building layer(s) are in principle identical, at least two of the three parameters - those in the plane of the layer - are identical. As in the case of triperiodic epitaxy (Royer, 1928, 1954), a threedimensional common sublattice exists (allotwin lattice), whose cell is based on the two common parameters in the plane of the layer, the third one being the shortest parameter, outside the plane of the layer, common to the cells of both individuals.

The allotwin laws are the twin laws for each of the individuals building the allotwin, to which the symmetry operations of the crystal(s) point group(s) have to be added. By indicating the first individual with a superscript and the second one with a subscript, the allotwin $Z_{T}={ }_{3}^{3}$ has to be considered too, whereas the $Z_{T}=33$ twin simply corresponds to a parallel intergrowth. Therefore, the number of possible laws becomes higher and depends upon the number of different polytypes undergoing allotwinning.

Since the geometrical appearance of the diffraction pattern of the $3 T$ polytype and of its twins is the same as (ideal) $1 M$ twinned as $Z_{T}=351$ or 3451 (see $\S 6.4$ ), the contribution from $3 T$ does not produce any independent pattern: it is not taken into account in the following systematic analysis.

The three basic monoclinic polytypes can produce three binary (two-portion) allotwins $\left(1 M-2 M_{1} ; 1 M-2 M_{2} ; 2 M_{1}-2 M_{2}\right)$ and one ternary (three-portion) allotwin $\left(1 M-2 M_{1}-2 M_{2}\right)$. Binary $(A B)$ and ternary $(A B C)$ allotwins are henceforth indicated by ${ }_{B}{ }_{B}$ and ${ }^{A} B_{C}$, respectively, where $A, B$ and $C$ represent the $Z_{T}$ symbols for each portion of the allotwin.

All these can be described on the following basis:

(i) The allotwin can be thought of as built up by two (binary allotwin) or three (ternary allotwin) portions $(A, B, C)$, each consisting only of individuals of the same polytype, which in their turn can be twinned; $1 M$ is taken as the first portion $(A)$ of the allotwin; when $1 M$ is not involved (binary allotwin $2 M_{1}-2 M_{2}$ ), the portion $A$ is $2 M_{1}$.

(ii) Since the individuals building the twin or allotwin are related by point-group operations, the $A-B-C$ sequence has no influence on the composite lattice and the two or three portions can be described as juxtaposed and non-mixed; for example, $Z_{T}={ }_{4}^{3}{ }_{6}^{5}$ is equivalent to $Z_{T}={ }^{35}{ }_{46}$.

(iii) Within each single portion $(A, B, C)$, the restrictions on the possible orientations derived for the twins are retained, but they are not applicable when comparing individuals belonging to different portions.

(iv) The first individual of the first portion $(A)$ is fixed in the orientation $Z_{T}=3$, but this restriction is not applicable for the first individual of the other portions. Therefore, the number of possible orientations for $B$ and $C$ portions has to be multiplied by the number of independent orientations of the minimal rhombus, as determined by the limiting symmetry ( $\$ 3)$, namely six for $2 M_{1}$ and two for $2 M_{2}$.

The minimal rhombi of the allotwins are calculated as combinations of the minimal rhombi of each portion, but the number of minimal rhombi to be considered depends upon the limiting symmetry. The minimal rhombi of two twins of $1 M$ which are equivalent through an $n \times 60^{\circ}$ rotation about the normal to (001) can produce two independent minimal rhombi when combined with a minimal rhombus of $2 M_{1}$. Therefore, in the derivation of the reciprocal lattice of $1 M-2 M_{1}$ allotwin, for both polytypes the minimal rhombi of all the 31 twins in Table 2 have to be considered. To these, the minimal rhombus corresponding to the single crystal has to be added. Moreover, if the minimal rhombi of $1 M$ (first individual in orientation $Z_{T}=3$ ) are kept fixed, the six independent orientations of each of the 32 minimal rhombi of $2 M_{1}$ have to be considered. For the $2 M_{2}$ polytype, there are only two independent orientations of the individual w.r.l. $\left(Z_{T}=U\right.$ or $\left.Z_{T}=E\right)$ and only one for the twin reciprocal lattice $\left(Z_{T}=U E\right)$. In deriving the reciprocal lattice of $1 M-2 M_{2}$ or $2 M_{1}-2 M_{2}$ allotwins, for Class a polytypes, only the minimal rhombus of the single-crystal and the minimal rhombi of the 23 twins related by $(2 n+1) \times 60^{\circ}$ rotations have to be combined with the three $(U, E, U E)$ minimal rhombi of $2 M_{2}$. The remaining eight minimal rhombi of Class a polytypes are related to some of the other 23 by $2 n \times 60^{\circ}$ rotations, which are symmetry operations for the minimal rhombi of $2 M_{2}$ and cannot produce any further independent allotwin minimal rhombus. Finally, for the ternary allotwins $1 M-2 M_{1}-2 M_{2}$, the independent minimal rhombi of the binary allotwin $1 M-2 M_{1}$, and those related by $(2 n+1) \times 60^{\circ}$ rotations, have to be combined with the three minimal rhombi of $2 M_{2}$.

For each combination, the composite minimal rhombus obtained in this way, rotated by $n \times 60^{\circ}(0 \leq n \leq 5)$ and - for each of these rotations - reflected across (010), is compared with the ones calculated for the previous combinations and, if equivalent, is discarded. Because of their relatively high number, the resulting minimal rhombi for allotwins are shown in table format rather than in figure format. The number of independent minimal rhombi is $46\left(1 M-2 M_{1}\right.$ : Table 7$), 13$ $\left(1 M-2 M_{2}\right.$ : Table 8$), 15\left(2 M_{1}-2 M_{2}\right.$ : Table 9$)$ and $36\left(1 M-2 M_{1}-\right.$ $2 M_{2}$ : Table 10).

\section{Effect of the violation of the additional reflection conditions}

The above derivation of the composite reciprocal lattice of twins and allotwins has been obtained within the trigonal model. In the real structure, other distortions besides the trigonal rotation of the tetrahedra appear, which in principle result in the violation of the non-space-group absences of the family reflections and in additional weak reflections in the family rows. These are visible in dioctahedral and $\mathrm{Li}-\mathrm{Fe}$ micas (Rieder, 1968, 1970) and may help to distinguish some patterns that are equivalent within the trigonal model. The $D$-type $R_{i}$ of subfamily $A$ polytypes are either $1_{2}$ or $1_{3}$ and the $D$-type $C_{i}$ can be either $1_{2}-1_{3}$ or $2_{6}$. No weak reflection appears 
Table 7

Distribution of $I_{j}$ on the nine $C_{i}$ for the 46 distinguishable patterns of $1 M-2 M_{1}$ binary allotwins.

For the definition of $I_{j}$ and $C_{i}$, see Table 3 and $\S 6$, respectively ( $c f$. also Figs. 5 and 6).

\begin{tabular}{|c|c|c|c|c|c|c|c|c|}
\hline Allotwin & $D_{1}$ & $D_{2}$ & $X_{1}$ & $X_{2}$ & $X_{3}$ & $X_{4}$ & $X_{5}$ & $X_{6}$ \\
\hline $3_{3}$ & $2{ }_{6}$ & $2_{6}$ & $3_{6}$ & $3_{7}$ & $2{ }_{3}$ & $3_{6}$ & $3_{7}$ & $2{ }_{3}$ \\
\hline 3 & $1_{2}$ & $1_{3}$ & $3_{3}$ & $3_{5}$ & $3_{2}$ & $3_{6}$ & $3_{7}$ & $3_{4}$ \\
\hline $3_{5}^{4}$ & 26 & 26 & 25 & 24 & $3_{2}$ & $3_{3}$ & $3_{5}$ & $3_{4}$ \\
\hline $\begin{array}{l}3 \\
6\end{array}$ & $1_{2}$ & $1_{3}$ & 25 & 24 & 23 & 25 & $2_{4}$ & $2_{3}$ \\
\hline $\begin{array}{l}0_{3} \\
34\end{array}$ & $2_{6}$ & 26 & $4_{1}$ & 45 & $3_{3}$ & $3_{6}$ & $3_{7}$ & $3_{5}$ \\
\hline 34 & 26 & 26 & $3_{4}$ & $3_{2}$ & $4_{1}$ & $3_{3}$ & 35 & $4_{5}$ \\
\hline 35 & 26 & 26 & $3_{6}$ & $3_{7}$ & $3_{3}$ & $4_{1}$ & $4_{5}$ & 35 \\
\hline 35 & $1_{2}$ & $1_{3}$ & $4_{4}$ & $4_{4}$ & $4_{1}$ & $4_{1}$ & $4_{5}$ & $4_{5}$ \\
\hline $\begin{array}{r}35 \\
1\end{array}$ & 26 & 26 & $4_{4}$ & $4_{4}$ & $3_{4}$ & $3_{4}$ & $3_{2}$ & $3_{2}$ \\
\hline $\begin{array}{l}36 \\
4\end{array}$ & 26 & $2_{6}$ & $4_{4}$ & $4_{4}$ & $3_{2}$ & $3_{6}$ & $3_{7}$ & $3_{4}$ \\
\hline 3 & $2_{6}$ & $2_{6}$ & $5_{1}$ & $5_{3}$ & $4_{2}$ & $3_{6}$ & $3_{7}$ & $4_{3}$ \\
\hline 34 & $2_{6}$ & 26 & $4_{3}$ & $4_{2}$ & $3_{2}$ & $5_{1}$ & $5_{3}$ & $3_{4}$ \\
\hline 35 & $2_{6}$ & 26 & 25 & 24 & $4_{2}$ & $4_{3}$ & $4_{2}$ & $4_{3}$ \\
\hline 3 & 26 & 26 & $4_{6}$ & $4_{6}$ & $4_{2}$ & $5_{1}$ & $5_{3}$ & 43 \\
\hline 346 & $1_{2}$ & $1_{3}$ & $4_{3}$ & $4_{2}$ & $4_{2}$ & $4_{6}$ & $4_{6}$ & $4_{3}$ \\
\hline $3_{51}$ & 26 & 26 & $4_{3}$ & $4_{2}$ & $5_{2}$ & $4_{3}$ & $4_{2}$ & $5_{2}$ \\
\hline 36 & $2{ }_{6}$ & $2{ }_{6}$ & $4_{6}$ & $4_{6}$ & 23 & $4_{6}$ & $4_{6}$ & 23 \\
\hline 3 & 26 & 26 & $3_{3}$ & $3_{5}$ & $5_{2}$ & $4_{6}$ & $4_{6}$ & $5_{2}$ \\
\hline $\begin{array}{l}3 \\
42\end{array}$ & $1_{2}$ & $1_{3}$ & $5_{1}$ & $5_{3}$ & $5_{2}$ & $5_{1}$ & $5_{3}$ & $5_{2}$ \\
\hline $\begin{array}{r}345 \\
3\end{array}$ & 26 & 26 & $4_{1}$ & $4_{5}$ & $3_{3}$ & $4_{1}$ & $4_{5}$ & $3_{5}$ \\
\hline $\begin{array}{r}345 \\
4\end{array}$ & $2_{6}$ & 26 & $4_{4}$ & $4_{4}$ & $4_{1}$ & $4_{1}$ & $4_{5}$ & $4_{5}$ \\
\hline $346_{4}^{\circ}$ & $2_{6}$ & 26 & $4_{4}$ & $4_{4}$ & $4_{1}$ & $3_{6}$ & $3_{7}$ & $4_{5}$ \\
\hline $346_{2}^{4}$ & 26 & 26 & $4_{1}$ & $4_{5}$ & $3_{4}$ & $4_{4}$ & $4_{4}$ & $3_{2}$ \\
\hline 34 & $2{ }_{6}$ & $2{ }_{6}$ & $5_{1}$ & $5_{3}$ & $5_{1}$ & $3_{6}$ & $3_{7}$ & $5_{3}$ \\
\hline $34{ }_{45}$ & 26 & 26 & $4_{3}$ & $4_{2}$ & $4_{1}$ & $5_{1}$ & $5_{3}$ & $4_{5}$ \\
\hline 34 & $2_{6}$ & $2_{6}$ & $5_{2}$ & $5_{2}$ & $5_{1}$ & $5_{1}$ & $5_{3}$ & $5_{3}$ \\
\hline $34{ }_{52}$ & 26 & 26 & $5_{2}$ & $5_{2}$ & $5_{2}$ & $3_{3}$ & $3_{5}$ & $5_{2}$ \\
\hline 35 & $2_{6}$ & 26 & $5_{1}$ & $5_{3}$ & $5_{1}$ & $4_{1}$ & $4_{5}$ & $5_{3}$ \\
\hline 35 & 26 & 26 & $4_{6}$ & $4_{6}$ & $5_{1}$ & $5_{1}$ & $5_{3}$ & $5_{3}$ \\
\hline 35 & $2_{6}$ & 26 & $5_{3}$ & $5_{1}$ & $5_{2}$ & $4_{3}$ & $4_{2}$ & $5_{2}$ \\
\hline 35 & $2_{6}$ & $2_{6}$ & $4_{4}$ & $4_{4}$ & $5_{2}$ & $5_{2}$ & $5_{2}$ & $5_{2}$ \\
\hline 3645 & $2_{6}$ & 26 & $5_{3}$ & $5_{1}$ & $3_{2}$ & $5_{1}$ & $5_{3}$ & $3_{4}$ \\
\hline 36 & $2{ }_{6}$ & $2{ }_{6}$ & $4_{4}$ & $4_{4}$ & $5_{2}$ & $4_{6}$ & $4_{6}$ & $5_{2}$ \\
\hline $3_{345}$ & $2_{6}$ & 26 & $6_{1}$ & $6_{1}$ & $4_{2}$ & $5_{1}$ & $5_{3}$ & $4_{3}$ \\
\hline $\begin{array}{l}3 \\
346\end{array}$ & $2_{6}$ & $2{ }_{6}$ & $6_{1}$ & $6_{1}$ & $4_{2}$ & $4_{6}$ & $4_{6}$ & $4_{3}$ \\
\hline $\begin{array}{l}3 \\
341\end{array}$ & 26 & 26 & $5_{1}$ & $5_{3}$ & $6_{1}$ & $4_{6}$ & $4_{6}$ & $6_{1}$ \\
\hline 342 & $2_{6}$ & $2{ }_{6}$ & $5_{1}$ & $5_{3}$ & $6_{1}$ & $5_{1}$ & $5_{3}$ & $6_{1}$ \\
\hline 3 & 26 & 26 & $6_{1}$ & $6_{1}$ & $6_{1}$ & $6_{1}$ & $6_{1}$ & $6_{1}$ \\
\hline 3456 & $2{ }_{6}$ & $2{ }_{6}$ & $4_{3}$ & $4_{2}$ & $4_{2}$ & $6_{1}$ & $6_{1}$ & $4_{3}$ \\
\hline $\begin{array}{l}3 \\
451\end{array}$ & $2_{6}$ & $2_{6}$ & $4_{3}$ & $4_{2}$ & $5_{2}$ & $6_{1}$ & $6_{1}$ & $5_{2}$ \\
\hline 3452 & $2{ }_{6}$ & $2{ }_{6}$ & $6_{1}$ & $6_{1}$ & $5_{2}$ & $5_{1}$ & $5_{3}$ & $5_{2}$ \\
\hline $3_{462}^{3}$ & $1_{2}$ & $1_{3}$ & $6_{1}$ & $6_{1}$ & $6_{1}$ & $6_{1}$ & $6_{1}$ & $6_{1}$ \\
\hline $\begin{array}{l}3 \\
3456\end{array}$ & $2_{6}$ & $2_{6}$ & $6_{1}$ & $6_{1}$ & $4_{2}$ & $6_{1}$ & $6_{1}$ & $4_{3}$ \\
\hline $\begin{array}{l}3 \\
3452\end{array}$ & $2{ }_{6}$ & $2{ }_{6}$ & $6_{1}$ & $6_{1}$ & $6_{1}$ & $5_{1}$ & $5_{3}$ & $6_{1}$ \\
\hline 33461 & $2{ }_{6}$ & $2{ }_{6}$ & $6_{1}$ & $6_{1}$ & $6_{1}$ & $4_{6}$ & $4_{6}$ & $6_{1}$ \\
\hline $3_{4512}$ & $2{ }_{6}$ & $2{ }_{6}$ & $6_{1}$ & $6_{1}$ & $5_{2}$ & $6_{1}$ & $6_{1}$ & $5_{2}$ \\
\hline
\end{tabular}

in the case of the $1 M$ polytype. For the $2 M_{1}$ polytype, one weak reflection is expected at $l_{T}=5\left(D_{2}\right)$ or $l_{T}=4\left(D_{1}\right)$, transforming $1_{2}, 1_{3}$ and $2_{6}$ into $2_{5}, 2_{4}$ and $4_{6}$, respectively. For the $3 T$ polytype, two weak reflections are expected at $l_{T}=0,2\left(D_{2}\right)$ or $l_{T}=0,4\left(D_{1}\right)$, transforming both $1_{2}$ and $1_{3}$ into $3_{1}$. Composite patterns that are equivalent within the trigonal model can be in principle distinguished as follows.

(i) $1 M$ twinned as $Z_{T}=351$ vs $3 T$ untwinned or twinned by complete merohedry $\left(Z_{T}=U U^{\bullet}\right)$. Two weak reflections are expected on both the $D$-type $R_{i}$ and $C_{i}$ when the $3 T$ polytype is involved.

(ii) $1 M$ twinned as $Z_{T}=3451$ vs $3 T$ twinned by selective merohedry (involving both $Z_{T}=U$ and $Z_{T}=E$ ). One weak
Table 8

Distribution of $I_{j}$ on the nine $C_{i}$ for the 16 distinguishable patterns of $1 M-2 M_{2}$ binary allotwins.

For the definition of $I_{j}$ and $C_{i}$, see Table 3 and $\S 6$, respectively ( $c f$. also Figs. 5 and 6).

\begin{tabular}{lllllllll}
\hline Allotwin & $D_{1}$ & $D_{2}$ & $X_{1}$ & $X_{2}$ & $X_{3}$ & $X_{4}$ & $X_{5}$ & $X_{6}$ \\
\hline${ }^{3}{ }_{U}$ & $3_{3}$ & $3_{5}$ & $3_{6}$ & $3_{7}$ & $3_{2}$ & $2_{5}$ & $2_{4}$ & $3_{4}$ \\
${ }^{34}{ }^{U}$ & $4_{4}$ & $4_{4}$ & $4_{1}$ & $4_{5}$ & $4_{1}$ & $2_{5}$ & $2_{4}$ & $4_{5}$ \\
${ }^{35}{ }^{U}$ & $3_{3}$ & $3_{5}$ & $3_{6}$ & $3_{7}$ & $4_{1}$ & $3_{4}$ & $3_{2}$ & $4_{5}$ \\
${ }^{36}{ }^{U}$ & $4_{4}$ & $4_{4}$ & $3_{6}$ & $3_{7}$ & $3_{2}$ & $3_{7}$ & $3_{6}$ & $3_{4}$ \\
${ }^{32}{ }^{U}$ & $4_{4}$ & $4_{4}$ & $3_{6}$ & $3_{7}$ & $3_{2}$ & $3_{4}$ & $3_{2}$ & $3_{4}$ \\
${ }^{345}{ }^{U}$ & $4_{4}$ & $4_{4}$ & $4_{1}$ & $4_{5}$ & $4_{1}$ & $3_{4}$ & $3_{2}$ & $4_{5}$ \\
${ }^{346}{ }^{U}$ & $4_{4}$ & $4_{4}$ & $4_{1}$ & $4_{5}$ & $4_{1}$ & $3_{7}$ & $3_{6}$ & $4_{5}$ \\
${ }^{351}{ }^{U}$ & $3_{3}$ & $3_{5}$ & $4_{1}$ & $4_{5}$ & $4_{1}$ & $4_{5}$ & $4_{1}$ & $4_{5}$ \\
${ }^{352}{ }_{U}$ & $4_{4}$ & $4_{4}$ & $3_{6}$ & $3_{7}$ & $4_{1}$ & $3_{4}$ & $3_{2}$ & $4_{5}$ \\
${ }^{3456}{ }^{3}$ & $4_{4}$ & $4_{4}$ & $4_{1}$ & $4_{5}$ & $4_{1}$ & $4_{5}$ & $4_{1}$ & $4_{5}$ \\
${ }_{U E}^{34}$ & $3_{3}$ & $3_{5}$ & $4_{6}$ & $4_{6}$ & $5_{2}$ & $4_{6}$ & $4_{6}$ & $5_{2}$ \\
${ }^{35}{ }^{U E}$ & $4_{4}$ & $4_{4}$ & $5_{2}$ & $5_{2}$ & $5_{2}$ & $4_{6}$ & $4_{6}$ & $5_{2}$ \\
${ }^{36}{ }_{U E}^{U E}$ & $3_{3}$ & $3_{5}$ & $4_{6}$ & $4_{6}$ & $5_{2}$ & $5_{2}$ & $5_{2}$ & $5_{2}$ \\
${ }_{345}$ & $4_{4}$ & $4_{4}$ & $4_{6}$ & $4_{6}$ & $5_{2}$ & $4_{6}$ & $4_{6}$ & $5_{2}$ \\
${ }^{351}{ }_{U E}^{U E}$ & $4_{4}$ & $4_{4}$ & $5_{2}$ & $5_{2}$ & $5_{2}$ & $5_{2}$ & $5_{2}$ & $5_{2}$ \\
\hline
\end{tabular}

reflection corresponding to $l_{T}=0$ is expected on both the $D$-type $C_{i}$ when the $3 T$ polytype is involved.

(iii) $2 M_{1}-3 T$ allotwins vs $1 M-2 M_{1}$ allotwins. In both cases, on the $D$ rows one weak reflection, corresponding either to $l_{T}=1$ or to $l_{T}=5$, is expected from the $2 M_{1}$ individual contribution. If $3 T$ is involved, another weak reflection, corresponding to $l_{T}=0$, is expected, whereas if $1 \mathrm{M}$ is involved it does not appear. It is never possible to distinguish between a $2 M_{1}-3 T$ binary allotwin and a $1 M-2 M_{1}-3 T$ ternary allotwin.

(iv) $2 M_{2}-T$ allotwin $v s 1 M-2 M_{2}$ allotwin. If the $3 T$ contribution is from a single crystal $\left(Z_{T}=U\right.$ or $\left.Z_{T}=E\right)$ or from a twin by complete merohedry $\left(Z_{T}=U U^{\bullet}\right)$, the two $D$-type $C_{i}$ rows are $3_{3}$ and $3_{5}$ in the trigonal model, and the distinction is possible on the basis of the appearance of a weak reflection alternatively corresponding to $l_{T}=4$ and $l_{T}=2$. However, if the $3 T$ contribution comes from a twin by selective merohedry (involving thus both $Z_{T}=U$ and $Z_{T}=E$ ), no distinction is possible, since all the three reflections corresponding to $l_{T}=0,2$ and 4 are present on the two $D$ rows. The ternary $1 M-2 M_{2}-3 T$ allotwin cannot be distinguished, since the $1 M$ features are hidden by the $3 T$ ones.

\section{Discussion}

In this paper, it has been shown that the geometrical analysis of the reciprocal lattice permits one to derive and identify the relative rotations in twins or allotwins of mica polytypes in the case of twinning or allotwinning by reticular pseudo-merohedry and by selective merohedry. The analysis has been devoted to the four basic polytypes, but can be easily extended to other polytypes, by redefining the period along $\mathbf{c}^{*}$ of the 'twin setting'. When dealing with a single polytype, the twin setting is best defined to coincide with the $C_{1}$ setting, which is based on the cell of the twin lattice. The adoption of a twin 
Table 9

Distribution of $I_{j}$ on the nine $C_{i}$ for the 13 distinguishable patterns of $2 M_{1}-2 M_{2}$ binary allotwins.

For the definition of $I_{j}$ and $C_{i}$, see Table 3 and $\S 6$, respectively ( $c f$. also Figs. 5 and 6).

\begin{tabular}{lllllllll}
\hline Allotwin & $D_{1}$ & $D_{2}$ & $X_{1}$ & $X_{2}$ & $X_{3}$ & $X_{4}$ & $X_{5}$ & $X_{6}$ \\
\hline${ }^{3}{ }_{U}$ & $3_{5}$ & $3_{3}$ & $2_{4}$ & $2_{5}$ & $4_{2}$ & $4_{6}$ & $4_{6}$ & $4_{3}$ \\
${ }^{34}{ }^{U}$ & $4_{4}$ & $4_{4}$ & $4_{2}$ & $4_{3}$ & $4_{2}$ & $4_{6}$ & $4_{6}$ & $4_{3}$ \\
${ }^{35}{ }^{U}$ & $3_{5}$ & $3_{3}$ & $4_{6}$ & $4_{6}$ & $4_{2}$ & $6_{1}$ & $6_{1}$ & $4_{3}$ \\
${ }^{36}$ & $4_{4}$ & $4_{4}$ & $4_{6}$ & $4_{6}$ & $4_{2}$ & $4_{6}$ & $4_{6}$ & $4_{3}$ \\
${ }^{32}{ }^{U}$ & $4_{4}$ & $4_{4}$ & $2_{4}$ & $2_{5}$ & $6_{1}$ & $6_{1}$ & $6_{1}$ & $6_{1}$ \\
${ }^{345}{ }^{U}$ & $4_{4}$ & $4_{4}$ & $6_{1}$ & $6_{1}$ & $4_{2}$ & $6_{1}$ & $6_{1}$ & $4_{3}$ \\
${ }^{346}{ }^{U}$ & $4_{4}$ & $4_{4}$ & $6_{1}$ & $6_{1}$ & $4_{2}$ & $4_{6}$ & $4_{6}$ & $4_{3}$ \\
${ }^{351}{ }^{3}{ }^{35}$ & $3_{5}$ & $3_{3}$ & $6_{1}$ & $6_{1}$ & $6_{1}$ & $6_{1}$ & $6_{1}$ & $6_{1}$ \\
${ }^{3451}$ & $4_{4}$ & $4_{4}$ & $4_{6}$ & $4_{6}$ & $6_{1}$ & $6_{1}$ & $6_{1}$ & $6_{1}$ \\
${ }^{3}{ }^{U E}$ & $4_{4}$ & $4_{4}$ & $6_{1}$ & $6_{1}$ & $6_{1}$ & $6_{1}$ & $6_{1}$ & $6_{1}$ \\
${ }^{35}{ }^{U E}$ & $3_{5}$ & $3_{3}$ & $4_{6}$ & $4_{6}$ & $6_{1}$ & $4_{6}$ & $4_{6}$ & $6_{1}$ \\
${ }^{36}{ }_{U E}$ & $3_{5}$ & $3_{3}$ & $4_{6}$ & $4_{6}$ & $6_{1}$ & $6_{1}$ & $6_{1}$ & $6_{1}$ \\
\hline
\end{tabular}

setting with a period along $\mathbf{c}^{*}$ different from that of the $C_{1}$ setting is useful to compare the geometry of the reciprocal lattice of polytypes with different period. In such a case, the twin setting is best defined to have the shortest period along $\mathbf{c}^{*}$ in the $C_{1}$ setting among all the polytypes considered.

In the twin or allotwin lattices, nodes on planes parallel to (001) are either completely overlapped (twinning by merohedry or pseudo-merohedry, twin index 1) or are overlapped on one plane out of three (twinning by reticular pseudomerohedry, twin index 3). Other oriented crystal associations are known, in which complete restoration of the lattice nodes is not achieved in any plane. These oriented crystal associations are called 'plesiotwins' (Nespolo, Ferraris, Takeda \& Takéuchi, 1999) and are built on larger hexagonal cells based on multiple two-dimensional meshes in the (001) plane. The plesiotwin elements are twofold axes in the plane of the layer with higher indices; the corresponding rotations about the normal to (001) express the relative rotations between individuals in a plesiotwin and are operations belonging to noncrystallographic point groups, even neglecting the angular deviation from orthohexagonality $\varepsilon$. On the other contrary, the relative rotations between individuals in both twins and allotwins belong to crystallographic point groups (neglecting $\varepsilon)$. The derivation of the plesiotwin laws can be performed following the same procedure shown above for the twin laws. However, the row features no longer correspond to the regular tessellation $\{3,6\}$, but to a compound tessellation $\{3,6\}[n\{3,6\}]$, where $n$ is the multiplicity of the larger mesh in the (001) plane (Takeda \& Donnay, 1965). Only a subset of the individual reciprocal-lattice rows, $R_{i}$, namely one out of $n$, overlaps in composite rows $C_{i}$. The diffraction pattern from the $(h k 0)$ plane thus shows a complex superstructure, corresponding to the overlap of two (eventually more) lattices rotated about the normal (Rang, 1958; Zvyagin \& Gorshkov, 1966; Takéuchi et al., 1972; Sunagawa \& Tomura, 1976), which can be interpreted by the geometrical theories of the coincidence-site lattice (e.g. Santoro \& Mighell, 1973), whereas, in the case of twins and allotwins, the diffraction pattern from the
Table 10

Distribution of $I_{j}$ on the nine $C_{i}$ for the 36 distinguishable patterns of $1 M-2 M_{1}-2 M_{2}$ ternary allotwins.

For the definition of $I_{j}$ and $C_{i}$, see Table 3 and $\S 6$, respectively ( $c f$. also Figs. 5 and 6).

\begin{tabular}{|c|c|c|c|c|c|c|c|c|}
\hline Allotwin & $D_{1}$ & $D_{2}$ & $X_{1}$ & $X_{2}$ & $X_{3}$ & $X_{4}$ & $X_{5}$ & $X_{6}$ \\
\hline${ }^{3} 3_{U}$ & $4_{4}$ & $4_{4}$ & $3_{6}$ & $3_{7}$ & $4_{2}$ & $4_{6}$ & $4_{6}$ & $4_{3}$ \\
\hline${ }^{3} 4_{U}$ & $3_{3}$ & $3_{5}$ & $5_{1}$ & $5_{3}$ & $3_{2}$ & $4_{6}$ & $4_{6}$ & $3_{4}$ \\
\hline${ }^{3} 4_{E}$ & $3_{3}$ & $3_{5}$ & $4_{3}$ & $4_{2}$ & $5_{2}$ & $3_{6}$ & $3_{7}$ & $5_{2}$ \\
\hline${ }^{3} 4_{U E}$ & $3_{3}$ & $3_{5}$ & $6_{1}$ & $6_{1}$ & $5_{2}$ & $4_{6}$ & $4_{6}$ & $5_{2}$ \\
\hline${ }^{3} 5_{U}$ & $4_{4}$ & $4_{4}$ & $4_{6}$ & $4_{6}$ & $3_{2}$ & $4_{3}$ & $4_{2}$ & $3_{4}$ \\
\hline${ }^{3} 5_{E}$ & $4_{4}$ & $4_{4}$ & 25 & 24 & $5_{2}$ & $5_{1}$ & $5_{3}$ & $5_{2}$ \\
\hline${ }^{3} 5_{U E}$ & $4_{4}$ & $4_{4}$ & $4_{6}$ & $4_{6}$ & $5_{2}$ & $6_{1}$ & $6_{1}$ & $5_{2}$ \\
\hline${ }^{34} 3_{U}$ & $4_{4}$ & $4_{4}$ & $4_{1}$ & $4_{5}$ & $5_{1}$ & $4_{6}$ & $4_{6}$ & $5_{3}$ \\
\hline${ }^{34} 3_{E}$ & $4_{4}$ & $4_{4}$ & $5_{2}$ & $5_{2}$ & $4_{3}$ & $3_{6}$ & $3_{7}$ & $4_{2}$ \\
\hline${ }^{34} 5_{U}$ & $4_{4}$ & $4_{4}$ & $5_{2}$ & $5_{2}$ & $4_{1}$ & $4_{3}$ & $4_{2}$ & $4_{5}$ \\
\hline${ }^{34} 5_{E}$ & $4_{4}$ & $4_{4}$ & $3_{4}$ & $3_{2}$ & $5_{2}$ & $5_{1}$ & $5_{3}$ & $5_{2}$ \\
\hline${ }^{34} 5_{U E}$ & $4_{4}$ & $4_{4}$ & $5_{2}$ & $5_{2}$ & $5_{2}$ & $6_{1}$ & $6_{1}$ & $5_{2}$ \\
\hline${ }^{35} 3_{U}$ & $4_{4}$ & $4_{4}$ & $3_{6}$ & $3_{7}$ & $5_{1}$ & $5_{2}$ & $5_{2}$ & $5_{3}$ \\
\hline${ }^{35} 3_{E}$ & $4_{4}$ & $4_{4}$ & $4_{6}$ & $4_{6}$ & $4_{3}$ & $4_{1}$ & $4_{5}$ & $4_{2}$ \\
\hline${ }^{35} 4_{U}$ & $3_{3}$ & $3_{5}$ & $5_{1}$ & $5_{3}$ & $4_{1}$ & $5_{2}$ & $5_{2}$ & $4_{5}$ \\
\hline${ }^{35} 4_{U E}$ & $3_{3}$ & $3_{5}$ & $6_{1}$ & $6_{1}$ & $5_{2}$ & $5_{2}$ & $5_{2}$ & $5_{2}$ \\
\hline${ }^{36} 4_{U}$ & $4_{4}$ & $4_{4}$ & $5_{1}$ & $5_{3}$ & $3_{2}$ & $4_{6}$ & $4_{6}$ & $3_{4}$ \\
\hline${ }^{3} 34_{U}$ & $4_{4}$ & $4_{4}$ & $5_{1}$ & $5_{3}$ & $4_{2}$ & $4_{6}$ & $4_{6}$ & $4_{3}$ \\
\hline${ }^{3} 34_{E}$ & $4_{4}$ & $4_{4}$ & $6_{1}$ & $6_{1}$ & $6_{1}$ & $3_{6}$ & $3_{7}$ & $6_{1}$ \\
\hline${ }^{3} 45_{U}$ & $4_{4}$ & $4_{4}$ & $6_{1}$ & $6_{1}$ & $3_{2}$ & $6_{1}$ & $6_{1}$ & $3_{4}$ \\
\hline${ }^{3} 45_{E}$ & $4_{4}$ & $4_{4}$ & $4_{3}$ & $4_{2}$ & $5_{2}$ & $5_{1}$ & $5_{3}$ & $5_{2}$ \\
\hline${ }^{3} 45_{U E}$ & $4_{4}$ & $4_{4}$ & $6_{1}$ & $6_{1}$ & $5_{2}$ & $6_{1}$ & $6_{1}$ & $5_{2}$ \\
\hline${ }^{3} 35_{E}$ & $4_{4}$ & $4_{4}$ & $4_{6}$ & $4_{6}$ & $6_{1}$ & $5_{1}$ & $5_{3}$ & $6_{1}$ \\
\hline${ }^{3} 51_{U}$ & $4_{4}$ & $4_{4}$ & $6_{1}$ & $6_{1}$ & $5_{2}$ & $4_{3}$ & $4_{2}$ & $5_{2}$ \\
\hline${ }^{3} 41_{U}$ & $4_{4}$ & $4_{4}$ & $5_{1}$ & $5_{3}$ & $5_{2}$ & $4_{6}$ & $4_{6}$ & $5_{2}$ \\
\hline${ }^{3} 41_{E}$ & $4_{4}$ & $4_{4}$ & $4_{3}$ & $4_{2}$ & $5_{2}$ & $4_{6}$ & $4_{6}$ & $5_{2}$ \\
\hline${ }^{3} 42_{U}$ & $3_{3}$ & $3_{5}$ & $5_{1}$ & $5_{3}$ & $5_{2}$ & $6_{1}$ & $6_{1}$ & $5_{2}$ \\
\hline${ }^{3} 42_{U E}$ & $3_{3}$ & $3_{5}$ & $6_{1}$ & $6_{1}$ & $5_{2}$ & $6_{1}$ & $6_{1}$ & $5_{2}$ \\
\hline${ }^{345} 3_{U}$ & $4_{4}$ & $4_{4}$ & $4_{1}$ & $4_{5}$ & $5_{1}$ & $5_{2}$ & $5_{2}$ & $5_{3}$ \\
\hline${ }^{34} 34_{U}$ & $4_{4}$ & $4_{4}$ & $5_{1}$ & $5_{3}$ & $5_{1}$ & $4_{6}$ & $4_{6}$ & $5_{3}$ \\
\hline${ }^{34} 45_{U}$ & $4_{4}$ & $4_{4}$ & $6_{1}$ & $6_{1}$ & $4_{1}$ & $6_{1}$ & $6_{1}$ & $4_{5}$ \\
\hline${ }^{34} 35_{U}$ & $4_{4}$ & $4_{4}$ & $5_{2}$ & $5_{2}$ & $5_{1}$ & $6_{1}$ & $6_{1}$ & $5_{3}$ \\
\hline${ }^{34} 52_{U}$ & $4_{4}$ & $4_{4}$ & $5_{2}$ & $5_{2}$ & $5_{2}$ & $4_{3}$ & $4_{2}$ & $5_{2}$ \\
\hline${ }^{34} 52_{E}$ & $4_{4}$ & $4_{4}$ & $5_{2}$ & $5_{2}$ & $5_{2}$ & $5_{1}$ & $5_{3}$ & $5_{2}$ \\
\hline${ }^{35} 34_{U}$ & $4_{4}$ & $4_{4}$ & $5_{1}$ & $5_{3}$ & $5_{1}$ & $5_{2}$ & $5_{2}$ & $5_{3}$ \\
\hline${ }^{3} 345_{E}$ & $4_{4}$ & $4_{4}$ & $6_{1}$ & $6_{1}$ & $6_{1}$ & $5_{1}$ & $5_{3}$ & $6_{1}$ \\
\hline
\end{tabular}

( $h k 0)$ plane shows the same pseudo-hexagonal symmetry of the single crystal. The coincidence-site lattice corresponding to the plesiotwin lattice is obtained by applying rotations that connect two $X$ rows on the same orbit but belonging to two different sets (Fig. 6, thin dashed lines). The minimal rhombus approach applies to both twins and allotwins, but not to plesiotwins.

This research has been partly developed at the Mineralogical Institute, University of Tokyo, during a Doctoral Program (MN, 1995-1998) supported by the Japanese Ministry of Education, Science and Culture. Part of this work (HT) has been undertaken at the Research Institute, Chiba Institute of Technology, and supported by the Tokyo Club Foundation. MN thanks Professor Boris B. Zvyagin (IGEM, RAS, Moscow), Professor Slavomil Durovič (Slovak Academy of Sciences, Bratislava) and Professor Vladimir Koptsik (University of Moscow) for profitable discussions, and Professor Masamichi Miyamoto (University of Tokyo) and 
Professor Yasunari Watanabe (Teikyo Heisei University) for their constant encouragement. The comments from two anonymous referees helped us in improving the clearness of the manuscript. GF acknowledges financial support from the Italian National Research Council (CNR) and from the Italian Ministry of University and of Scientific and Technological Research (MURST).

\section{References}

Abbott, R. N. Jr \& Burnham, C. W. (1988). Am. Mineral. 73, 105-118. Arnold, H. (1996). International Tables for Crystallography, Vol. A, 4th ed., edited by Th. Hahn, Section 5. Dordrecht: Kluwer Academic Publishers.

Babu, V. R. R. M. (1969). Indian Mineral. 9, 41-46.

Backhaus, K.-O. \& Durovič, S. (1984). Clays Clay Miner. 32, 453-463.

Baronnet, A. \& Kang, Z. C. (1989). Phase Transit. 16/17, 477-493.

Catti, M. \& Ferraris, G. (1976). Acta Cryst. A32, 163-165.

Curien, H. \& Donnay, J. D. H. (1959). Am. Mineral. 44, 1067-1070.

Curien, H. \& Le Corre, Y. (1958). Bull. Soc. Fr. Minéral. Cristallogr. 81, 126-132.

Donnay, G., Morimoto, N., Takeda, H. \& Donnay, J. D. H. (1964). Acta Cryst. 17, 1369-1373.

Dornberger-Schiff, K. (1959). Acta Cryst. 12, 173.

Dornberger-Schiff, K., Backhaus, K.-O. \& Durovič, S. (1982). Clays Clay Miner. 30, 364-374.

Dornberger-Schiff, K. \& Fichtner, K. (1972). Krist. Tech. 7, 1035-1056.

Ďurovič, S. (1994a). Ceramics-Silikáty, 38, 81-84.

Durovič, S. (1994b). In Aperiodic '94. Proceedings of the International Conference on Aperiodic Crystals, edited by G. Chapuis \& W. Paciorek, pp. 595-599. Singapore/New Jersey/London/Hong Kong: World Scientific.

Durovič, S. (1997). Modular Aspects of Minerals/EMU Notes in Mineralogy, Vol. 1, edited by S. Merlino, pp. 1-28. Budapest: Eötvös University Press.

Ďurovič, S. (1999). International Tables for Crystallography, Vol. C, 2nd ed., edited by A. C. J. Wilson \& E. Prince, Section 9.2.2. Dordrecht: Kluwer Academic Publishers.

Ďurovič, S. \& Weiss, Z. (1986). Bull. Minéral. 109, 15-29.

Ďurovič, S., Weiss, Z. \& Backhaus, K.-O. (1984). Clays Clay Miner. 32, 454-474.

Fichtner, K. (1977). Beitr. Alg. Geom. 6, 71-99.

Friedel, G. (1904). Étude sur les Groupements Cristallins. Extract from Bullettin de la Société de l'Industrie Minérale, 4th series, Vols. III and IV. Saint-Étienne: Société de l'Imprimerie Théolier J. Thomas et C.

Friedel, G. (1926). Leçons de Cristallographie. Nancy/Paris/Strasbourg: Berger-Levrault.

Giacovazzo, C. (1992). Fundamentals of Crystallography, edited by C. Giacovazzo, pp. 1-60. IUCr/Oxford University Press,.

Goldschmidt, V. (1918). Atlas der Krystallformen, Band IV. Heidelberg: Carl Winters Universitätsbuchhandlung,

Gresens, R. L. \& Stensrud, H. L. (1971). Lithos, 4, 63-69.

Guinier, A., Bokij, G. B., Boll-Dornberger, K., Cowley, J. M., Ďurovič, S., Jagodzinski, H., Khrisna, P., de Wolff, P. M., Zvyagin, B. B., Cox, D. E., Goodman, P., Hahn, Th., Kuchitsu, K. \& Abrahams, S. C. (1984). Acta Cryst. A40, 399-404.

Hurlbut, C. S. (1956). Am. Mineral. 41, 892-898.
Kogure, T. (1997). Mineral. J. 19, 155-164.

Kogure, T. \& Nespolo, M. (1999). Acta Cryst. B55, 507-516.

Merlino, S. (1990). Per. Mineral. 59, 69-92.

Mügge, O. (1898). Neues Jahrb. Mineral. Geol. Paläontol. 1, 71-158.

Nespolo, M. (1999a). Mineral. J. 21, 53-85.

Nespolo, M. (1999b). J. Teikyo Heisei Univ. 11, 1-16.

Nespolo, M. \& Ferraris, G. (2000). Z. Kristallogr. 215. In the press.

Nespolo, M., Ferraris, G. \& Durović, S. (1999). Z. Kristallogr. 214, 776-779.

Nespolo, M., Ferraris, G., Takeda, H. \& Takéuchi, Y. (1999). Z. Kristallogr. 214, 378-382.

Nespolo, M. \& Kogure, T. (1998). Z. Kristallogr. 213, 4-12.

Nespolo, M., Kogure, T. \& Ferraris, G. (1999). Z. Kristallogr. 214, 5-8.

Nespolo, M., Kogure, T., Takeda, H. \& Ferraris, G. (1999). Acta Cryst. A55, C214.

Nespolo, M. \& Takeda, H. (1999). Mineral. J. 21, 103-118.

Nespolo, M., Takeda, H. \& Ferraris, G. (1997). Modular Aspects of Minerals/EMU Notes in Mineralogy, Vol. 1, edited by S. Merlino, pp. 81-118. Budapest: Eötvös University Press.

Nespolo, M., Takeda, H. \& Ferraris, G. (1998). Acta Cryst. A54, 348-356.

Nespolo, M., Takeda, H., Ferraris, G. \& Kogure, T. (1997). Mineral. J. 19, 173-186.

Nespolo, M., Takeda, H., Kogure, T. \& Ferraris, G. (1999). Acta Cryst. A55, 659-676.

Pabst, A. (1955). Am. Mineral. 40, 967-974.

Pauling, L. (1930). Proc. Natl Acad. Sci. USA, 16, 123-129.

Peacock, M. A. \& Ferguson, R. B. (1943). Univ. Toronto, Stud. Mineral. 48, 65-82.

Rang, O (1958). Z. Phys. 152, 194-202.

Rieder, M. (1968). Science, 160, 1338-1340.

Rieder, M. (1970). Z. Kristallogr. 132, 161-184.

Ross, M., Takeda, H. \& Wones, D. R. (1966). Science, 151, 191-193.

Royer, L. (1928). Bull. Soc. Fr. Minéral. Cristallogr. 51, 7-159.

Royer, L. (1954). Bull. Soc. Fr. Minéral. Cristallogr. 77, 1004-1028.

Sadanaga, R., Sawada, T., Ohsumi, K. \& Kamiya, K. (1980). J. Jpn Assoc. Min. Petr. Econ. Geol. Spec. Issue, 2, 23-29.

Sadanaga, R. \& Takéuchi, Y. (1961). Z. Kristallogr. 116, 406-429.

Santoro, A. (1974). Acta Cryst. A30, 224-231.

Santoro, A. \& Mighell, A. D. (1972). Acta Cryst. A28, 284-287.

Santoro, A. \& Mighell, A. D. (1973). Acta Cryst. A29, 169-175.

Smith, J. V. \& Yoder, H. S. (1956). Mineral. Mag. 31, 209-235.

Sunagawa, I. (1964). Am. Mineral. 49, 1427-1434.

Sunagawa, I. \& Tomura, S. (1976). Am. Mineral. 61, 939-943.

Takano, Y. \& Takano, K. (1958). J. Mineral. Soc. Jpn, 3, 674-692. (In Japanese.)

Takeda, H. (1967). Acta Cryst. 22, 845-853.

Takeda, H. (1971). Am. Mineral. 56 1042-1056.

Takeda, H. \& Donnay, J. D. H. (1965). Acta Cryst. 19, 474-476.

Takeda, H. \& Ross, M. (1995). Am. Mineral. 80, 715-724.

Takéuchi, Y., Sadanaga, R. \& Aikawa, N. (1972). Z. Kristallogr. 136, 207-225.

Tatekawa, M. (1975). Mineral. J. 7, 575-581.

Weiss, Z. \& Wiewióra, A. (1986). Clays Clay Miner. 34, 53-68.

Zvyagin, B. B. (1997). Modular Aspects of Minerals/EMU Notes in Mineralogy, Vol. 1, edited by S. Merlino, pp. 345-372. Budapest: Eötvös University Press.

Zvyagin, B. B. \& Drits, V. A. (1996). Clays Clay Miner. 44, 297-303.

Zvyagin, B. B. \& Gorshkov, A. I. (1966). Sixth International Congress for Electron Microscopy, Kyoto, Japan. Electron Microscopy 1966, Vol. I, edited by R. Uyeda, pp. 603-604. Tokyo: Maruzen. 